\title{
Perturbation Theory Treatment of Spin-Orbit Coupling. I: Double Perturbation Theory Based on a Single-Reference Initial Approximation
}

\author{
Jacques K. Desmarais* \\ Dipartimento di Chimica, Università di Torino, via Giuria 5, 10125 Torino, Italy and \\ Université de Pau et des Pays de l'Adour, E2S UPPA, CNRS, IPREM, Pau, France \\ Alessandro Erba \\ Dipartimento di Chimica, Università di Torino, via Giuria 5, 10125 Torino, Italy \\ Jean-Pierre Flament \\ Université de Lille, CNRS, UMR 8523 - PhLAM - Physique des Lasers, Atomes et Molécules, 59000 Lille, France \\ Bernard Kirtman ${ }^{\dagger}$ \\ Department of Chemistry and Biochemistry, University of California, Santa Barbara, California 93106, USA
}

\section{DETAILS ON THE DERIVATION OF EQUATION 18 FOR $E^{(4,0)}$}

\section{A. The Singles Contribution $E_{S}^{(4,0)}$}

The expression for $E_{S}^{(4,0)}$ can be obtained from Eq. (A3) of the main text, by limiting the sums over configurations to only singly-excited determinants $(\mathrm{S})$ :

$$
\begin{aligned}
E_{S}^{(4,0)} & =\sum_{I J K}^{S} \frac{\left\langle\psi^{(0,0)}\left|\hat{h}_{S O}\right| \psi_{I}^{(0,0)}\right\rangle\left\langle\psi_{I}^{(0,0)}\left|\hat{h}_{S O}\right| \psi_{J}^{(0,0)}\right\rangle\left\langle\psi_{J}^{(0,0)}\left|\hat{h}_{S O}\right| \psi_{K}^{(0,0)}\right\rangle\left\langle\psi_{K}^{(0,0)}\left|\hat{h}_{S O}\right| \psi^{(0,0)}\right\rangle}{\left(E^{(0,0)}-E_{I}^{(0,0)}\right)\left(E^{(0,0)}-E_{J}^{(0,0)}\right)\left(E^{(0,0)}-E_{K}^{(0,0)}\right)} \\
& -E^{(2,0)} \sum_{I}^{S} \frac{\left\langle\psi^{(0,0)}\left|\hat{h}_{S O}\right| \psi_{I}^{(0,0)}\right\rangle\left\langle\psi_{I}^{(0,0)}\left|\hat{h}_{S O}\right| \psi^{(0,0)}\right\rangle}{\left(E^{(0,0)}-E_{I}^{(0,0)}\right)^{2}} .
\end{aligned}
$$

We can express $\left|\psi_{I}^{(0,0)}\right\rangle,\left|\psi_{J}^{(0,0)}\right\rangle$ and $\left|\psi_{K}^{(0,0)}\right\rangle$ as generic singly-excited determinants and write:

$$
\begin{aligned}
E_{S}^{(4,0)} & =\sum_{m n o}^{\text {occ }} \sum_{p q r}^{\text {virt }} \frac{\left\langle\psi^{(0,0)}\left|\hat{h}_{S O}\right| \psi_{m}^{p}\right\rangle\left\langle\psi_{m}^{p}\left|\hat{h}_{S O}\right| \psi_{n}^{q}\right\rangle\left\langle\psi_{n}^{q}\left|\hat{h}_{S O}\right| \psi_{o}^{r}\right\rangle\left\langle\psi_{o}^{r}\left|\hat{h}_{S O}\right| \psi^{(0,0)}\right\rangle}{\left(E^{(0,0)}-E_{m}^{p}\right)\left(E^{(0,0)}-E_{n}^{q}\right)\left(E^{(0,0)}-E_{o}^{r}\right)} \\
& -E^{(2,0)} \sum_{m}^{\text {occ }} \sum_{p}^{\text {virt }} \frac{\left\langle\psi^{(0,0)}\left|\hat{h}_{S O}\right| \psi_{m}^{p}\right\rangle\left\langle\psi_{m}^{p}\left|\hat{h}_{S O}\right| \psi^{(0,0)}\right\rangle}{\left(E^{(0,0)}-E_{m}^{p}\right)^{2}}
\end{aligned}
$$

We can now evaluate all integrals in Eq. (S2) using the Slater-Condon rules. For integrals involving singly-excited determinants and the SOC operator:

$$
\begin{aligned}
\left\langle\psi^{(0,0)}\left|\hat{h}_{S O}\right| \psi_{m}^{p}\right\rangle & =\left\langle m\left|\hat{h}_{S O}\right| p\right\rangle \\
\left\langle\psi_{n}^{q}\left|\hat{h}_{S O}\right| \psi_{o}^{r}\right\rangle & =\delta_{o, n}\left\langle q\left|\hat{h}_{S O}\right| r\right\rangle-\delta_{q, r}\left\langle o\left|\hat{h}_{S O}\right| n\right\rangle \\
\left\langle\psi_{o}^{r}\left|\hat{h}_{S O}\right| \psi^{(0,0)}\right\rangle & =\left\langle r\left|\hat{h}_{S O}\right| o\right\rangle
\end{aligned}
$$

Substituting Eqs. (S3a)-(S3c) in Eq. (S2) and using also the expression for $E^{(2,0)}$ in terms of singly-excited configurations, we

\footnotetext{
*Electronic address: jacqueskontak.desmarais@unito.it

${ }^{\dagger}$ Electronic address: kirtman@chem.ucsb.edu
} 
get:

$$
\begin{aligned}
E_{S}^{(4,0)} & =\sum_{m, n, o}^{o c c} \sum_{p, q, r}^{\text {virt }} \frac{\left\langle m\left|\hat{h}_{S O}\right| p\right\rangle\left\{\delta_{m, n}\left\langle p\left|\hat{h}_{S O}\right| q\right\rangle-\delta_{p, q}\left\langle n\left|\hat{h}_{S O}\right| m\right\rangle\right\}\left\{\delta_{o, n}\left\langle q\left|\hat{h}_{S O}\right| r\right\rangle-\delta_{q, r}\left\langle o\left|\hat{h}_{S O}\right| n\right\rangle\right\}\left\langle r\left|\hat{h}_{S O}\right| o\right\rangle}{\left(\varepsilon_{m}-\varepsilon_{p}\right)\left(\varepsilon_{n}-\varepsilon_{q}\right)\left(\varepsilon_{o}-\varepsilon_{r}\right)} \\
& -\sum_{m, n}^{o c c} \sum_{p, q}^{\text {virt }} \frac{\left\langle m\left|\hat{h}_{S O}\right| p\right\rangle\left\langle p\left|\hat{h}_{S O}\right| m\right\rangle\left\langle n\left|\hat{h}_{S O}\right| q\right\rangle\left\langle q\left|\hat{h}_{S O}\right| n\right\rangle}{\left(\varepsilon_{m}-\varepsilon_{p}\right)\left(\varepsilon_{m}-\varepsilon_{p}\right)\left(\varepsilon_{n}-\varepsilon_{q}\right)} .
\end{aligned}
$$

Evaluating the Kronecker deltas in Eq. (S4) gives:

$$
\begin{aligned}
E_{S}^{(4,0)} & =\sum_{m}^{o c c} \sum_{p, q, r}^{\text {virt }} \frac{\left\langle m\left|\hat{h}_{S O}\right| p\right\rangle\left\langle p\left|\hat{h}_{S O}\right| q\right\rangle\left\langle q\left|\hat{h}_{S O}\right| r\right\rangle\left\langle r\left|\hat{h}_{S O}\right| m\right\rangle}{\left(\varepsilon_{m}-\varepsilon_{p}\right)\left(\varepsilon_{m}-\varepsilon_{q}\right)\left(\varepsilon_{m}-\varepsilon_{r}\right)} \\
& -\sum_{m, n} \sum_{p, q}^{\text {occ }} \frac{\left\langle m\left|\hat{h}_{S O}\right| p\right\rangle\left\langle p\left|\hat{h}_{S O}\right| q\right\rangle\left\langle n\left|\hat{h}_{S O}\right| m\right\rangle\left\langle q\left|\hat{h}_{S O}\right| n\right\rangle}{\left(\varepsilon_{m}-\varepsilon_{p}\right)\left(\varepsilon_{n}-\varepsilon_{q}\right)\left(\varepsilon_{m}-\varepsilon_{q}\right)} \\
& -\sum_{m, n}^{o c c} \sum_{p, q}^{\text {virt }} \frac{\left\langle m\left|\hat{h}_{S O}\right| p\right\rangle\left\langle n\left|\hat{h}_{S O}\right| m\right\rangle\left\langle p\left|\hat{h}_{S O}\right| q\right\rangle\left\langle q\left|\hat{h}_{S O}\right| n\right\rangle}{\left(\varepsilon_{m}-\varepsilon_{p}\right)\left(\varepsilon_{n}-\varepsilon_{p}\right)\left(\varepsilon_{n}-\varepsilon_{q}\right)} \\
& +\sum_{m, n, o}^{o c c} \sum_{p}^{\text {virt }} \frac{\left\langle m\left|\hat{h}_{S O}\right| p\right\rangle\left\langle n\left|\hat{h}_{S O}\right| m\right\rangle\left\langle o\left|\hat{h}_{S O}\right| n\right\rangle\left\langle p\left|\hat{h}_{S O}\right| o\right\rangle}{\left(\varepsilon_{m}-\varepsilon_{p}\right)\left(\varepsilon_{n}-\varepsilon_{p}\right)\left(\varepsilon_{n}-\varepsilon_{q}\right)} \\
& -\sum_{m, n}^{o c c} \sum_{p, q}^{\text {virt }} \frac{\left\langle m\left|\hat{h}_{S O}\right| p\right\rangle\left\langle p\left|\hat{h}_{S O}\right| m\right\rangle\left\langle n\left|\hat{h}_{S O}\right| q\right\rangle\left\langle q\left|\hat{h}_{S O}\right| n\right\rangle}{\left(\varepsilon_{m}-\varepsilon_{p}\right)\left(\varepsilon_{m}-\varepsilon_{p}\right)\left(\varepsilon_{n}-\varepsilon_{q}\right)} .
\end{aligned}
$$

Then, a switch of the indices $m, p \leftrightarrow n, q$ in the third line of Eq. (S5) and combining it with the second line, we obtain:

$$
\begin{aligned}
E_{S}^{(4,0)} & =-\sum_{m, n}^{o c c} \sum_{p, q}^{\text {virt }} 2 R\left[\frac{\left\langle m\left|\hat{h}_{S O}\right| p\right\rangle\left\langle p\left|\hat{h}_{S O}\right| q\right\rangle\left\langle n\left|\hat{h}_{S O}\right| m\right\rangle\left\langle q\left|\hat{h}_{S O}\right| n\right\rangle}{\left(\varepsilon_{m}-\varepsilon_{p}\right)\left(\varepsilon_{n}-\varepsilon_{q}\right)\left(\varepsilon_{m}-\varepsilon_{q}\right)}\right] \\
& +\sum_{m}^{o c c} \sum_{p, q, r}^{\text {virt }} \frac{\left\langle m\left|\hat{h}_{S O}\right| p\right\rangle\left\langle p\left|\hat{h}_{S O}\right| q\right\rangle\left\langle q\left|\hat{h}_{S O}\right| r\right\rangle\left\langle r\left|\hat{h}_{S O}\right| m\right\rangle}{\left(\varepsilon_{m}-\varepsilon_{p}\right)\left(\varepsilon_{m}-\varepsilon_{q}\right)\left(\varepsilon_{m}-\varepsilon_{r}\right)} \\
& +\sum_{m, n, o}^{o c c} \sum_{p}^{\text {virt }} \frac{\left\langle m\left|\hat{h}_{S O}\right| p\right\rangle\left\langle n\left|\hat{h}_{S O}\right| m\right\rangle\left\langle o\left|\hat{h}_{S O}\right| n\right\rangle\left\langle p\left|\hat{h}_{S O}\right| o\right\rangle}{\left(\varepsilon_{m}-\varepsilon_{p}\right)\left(\varepsilon_{n}-\varepsilon_{p}\right)\left(\varepsilon_{o}-\varepsilon_{p}\right)} \\
& -\sum_{m, n}^{o c c} \sum_{p, q}^{\text {virt }} \frac{\left\langle m\left|\hat{h}_{S O}\right| p\right\rangle\left\langle p\left|\hat{h}_{S O}\right| m\right\rangle\left\langle n\left|\hat{h}_{S O}\right| q\right\rangle\left\langle q\left|\hat{h}_{S O}\right| n\right\rangle}{\left(\varepsilon_{m}-\varepsilon_{p}\right)^{2}\left(\varepsilon_{n}-\varepsilon_{q}\right)} .
\end{aligned}
$$

\section{B. The Doubles Contribution $E_{D}^{(4,0)}$}

We can write $E_{D}^{(4,0)}$ from Eq. (A3) of the main text by requiring that each term in the sum over configurations include at least one doubly-excited (D) configuration:

$$
E_{D}^{(4,0)}=\sum_{I J K}^{S D} \frac{\left\langle\psi^{(0,0)}\left|\hat{h}_{S O}\right| \psi_{I}^{(0,0)}\right\rangle\left\langle\psi_{I}^{(0,0)}\left|\hat{h}_{S O}\right| \psi_{J}^{(0,0)}\right\rangle\left\langle\psi_{J}^{(0,0)}\left|\hat{h}_{S O}\right| \psi_{K}^{(0,0)}\right\rangle\left\langle\psi_{K}^{(0,0)}\left|\hat{h}_{S O}\right| \psi^{(0,0)}\right\rangle}{\left(E^{(0,0)}-E_{I}^{(0,0)}\right)\left(E^{(0,0)}-E_{J}^{(0,0)}\right)\left(E^{(0,0)}-E_{K}^{(0,0)}\right)} .
$$

Because $\hat{h}_{S O}$ is a sum of mono-electronic operators, the matrix elements $\left\langle\psi^{(0,0)}\left|\hat{h}_{S O}\right| \psi_{I}^{(0,0)}\right\rangle$ and $\left\langle\psi_{K}^{(0,0)}\left|\hat{h}_{S O}\right| \psi^{(0,0)}\right\rangle$ in Eq. (S7) show us that $\left|\psi_{I}^{(0,0)}\right\rangle$ and $\left|\psi_{K}^{(0,0)}\right\rangle$ can only be singly-excited configurations. We must then conclude that $\left|\psi_{J}^{(0,0)}\right\rangle$ is a generic doubly-excited configuration, which gives:

$$
E_{D}^{(4,0)}=\sum_{l, o}^{o c c} \sum_{m>n}^{o c c} \sum_{p, s}^{\text {virt }} \sum_{q>r}^{v i r t} \frac{\left\langle\psi^{(0,0)}\left|\hat{h}_{S O}\right| \psi_{l}^{p}\right\rangle\left\langle\psi_{l}^{p}\left|\hat{h}_{S O}\right| \psi_{m n}^{q r}\right\rangle\left\langle\psi_{m n}^{q r}\left|\hat{h}_{S O}\right| \psi_{o}^{s}\right\rangle\left\langle\psi_{o}^{s}\left|\hat{h}_{S O}\right| \psi^{(0,0)}\right\rangle}{\left(E^{(0,0)}-E_{l}^{p}\right)\left(E^{(0,0)}-E_{m n}^{q r}\right)\left(E^{(0,0)}-E_{o}^{s}\right)} .
$$

We can evaluate the integrals involving both singly- and doubly-excited configurations, as well as the SOC operator as follows:

$$
\begin{aligned}
\left\langle\psi_{l}^{p}\left|\hat{h}_{S O}\right| \psi_{m n}^{q r}\right\rangle & =\delta_{p, q} \delta_{l, m}\left\langle n\left|\hat{h}_{S O}\right| r\right\rangle+\delta_{p, r} \delta_{l, n}\left\langle m\left|\hat{h}_{S O}\right| q\right\rangle-\delta_{p, q} \delta_{l, n}\left\langle m\left|\hat{h}_{S O}\right| r\right\rangle-\delta_{p, r} \delta_{l, m}\left\langle n\left|\hat{h}_{S O}\right| q\right\rangle \\
\left\langle\psi_{m n}^{q r}\left|\hat{h}_{S O}\right| \psi_{o}^{S}\right\rangle & =\delta_{s, q} \delta_{o, m}\left\langle r\left|\hat{h}_{S O}\right| n\right\rangle+\delta_{s, r} \delta_{o, n}\left\langle q\left|\hat{h}_{S O}\right| m\right\rangle-\delta_{s, q} \delta_{o, n}\left\langle r\left|\hat{h}_{S O}\right| m\right\rangle-\delta_{s, r} \delta_{o, m}\left\langle q\left|\hat{h}_{S O}\right| n\right\rangle .
\end{aligned}
$$


Substituting Eqs. (S9a) and (S9b) in Eq. (S8) yields:

$$
\begin{aligned}
E_{D}^{(4,0)} & =\sum_{l, o}^{o c c} \sum_{m>n}^{\text {occ }} \sum_{p, s}^{\text {virt }} \sum_{q>r}^{\text {virt }}\left\langle l\left|\hat{h}_{S O}\right| p\right\rangle\left\{\delta_{p, q} \delta_{l, m}\left\langle n\left|\hat{h}_{S O}\right| r\right\rangle+\delta_{p, r} \delta_{l, n}\left\langle m\left|\hat{h}_{S O}\right| q\right\rangle-\delta_{p, q} \delta_{l, n}\left\langle m\left|\hat{h}_{S O}\right| r\right\rangle-\delta_{p, r} \delta_{l, m}\left\langle n\left|\hat{h}_{S O}\right| q\right\rangle\right\} \\
& \times \frac{\left\{\delta_{s, q} \delta_{o, m}\left\langle r\left|\hat{h}_{S O}\right| n\right\rangle+\delta_{s, r} \delta_{o, n}\left\langle q\left|\hat{h}_{S O}\right| m\right\rangle-\delta_{s, q} \delta_{o, n}\left\langle r\left|\hat{h}_{S O}\right| m\right\rangle-\delta_{s, r} \delta_{o, m}\left\langle q\left|\hat{h}_{S O}\right| n\right\rangle\right\}\left\langle s\left|\hat{h}_{S O}\right| o\right\rangle}{\left(\varepsilon_{l}-\varepsilon_{p}\right)\left(\varepsilon_{m}+\varepsilon_{n}-\varepsilon_{q}-\varepsilon_{r}\right)\left(\varepsilon_{o}-\varepsilon_{s}\right)}
\end{aligned}
$$

Evaluating the Kronecker deltas in Eq. (S10) gives:

$$
\begin{aligned}
& E_{D}^{(4,0)}=\sum_{m>n}^{o c c} \sum_{q>r}^{\text {virt }} \frac{\left\langle m\left|\hat{h}_{S O}\right| q\right\rangle\left\langle n\left|\hat{h}_{S O}\right| r\right\rangle\left\langle r\left|\hat{h}_{S O}\right| n\right\rangle\left\langle q\left|\hat{h}_{S O}\right| m\right\rangle}{\left(\varepsilon_{m}-\varepsilon_{q}\right)\left(\varepsilon_{m}+\varepsilon_{n}-\varepsilon_{q}-\varepsilon_{r}\right)\left(\varepsilon_{m}-\varepsilon_{q}\right)} \\
& +\sum_{m>n}^{o c c} \sum_{q>r}^{\text {virt }} \frac{\left\langle m\left|\hat{h}_{S O}\right| q\right\rangle\left\langle n\left|\hat{h}_{S O}\right| r\right\rangle\left\langle q\left|\hat{h}_{S O}\right| m\right\rangle\left\langle r\left|\hat{h}_{S O}\right| n\right\rangle}{\left(\varepsilon_{m}-\varepsilon_{q}\right)\left(\varepsilon_{m}+\varepsilon_{n}-\varepsilon_{q}-\varepsilon_{r}\right)\left(\varepsilon_{n}-\varepsilon_{r}\right)} \\
& -\sum_{m>n}^{o c c} \sum_{q>r}^{v i r t} \frac{\left\langle m\left|\hat{h}_{S O}\right| q\right\rangle\left\langle n\left|\hat{h}_{S O}\right| r\right\rangle\left\langle r\left|\hat{h}_{S O}\right| m\right\rangle\left\langle q\left|\hat{h}_{S O}\right| n\right\rangle}{\left(\varepsilon_{m}-\varepsilon_{q}\right)\left(\varepsilon_{m}+\varepsilon_{n}-\varepsilon_{q}-\varepsilon_{r}\right)\left(\varepsilon_{n}-\varepsilon_{q}\right)} \\
& -\sum_{m>n}^{o c c} \sum_{q>r}^{v i r} \frac{\left\langle m\left|\hat{h}_{S O}\right| q\right\rangle\left\langle n\left|\hat{h}_{S O}\right| r\right\rangle\left\langle q\left|\hat{h}_{S O}\right| n\right\rangle\left\langle r\left|\hat{h}_{S O}\right| m\right\rangle}{\left(\varepsilon_{m}-\varepsilon_{q}\right)\left(\varepsilon_{m}+\varepsilon_{n}-\varepsilon_{q}-\varepsilon_{r}\right)\left(\varepsilon_{m}-\varepsilon_{r}\right)} \\
& +\sum_{m>n}^{o c c} \sum_{q>r}^{v i r} \frac{\left\langle n\left|\hat{h}_{S O}\right| r\right\rangle\left\langle m\left|\hat{h}_{S O}\right| q\right\rangle\left\langle r\left|\hat{h}_{S O}\right| n\right\rangle\left\langle q\left|\hat{h}_{S O}\right| m\right\rangle}{\left(\varepsilon_{n}-\varepsilon_{r}\right)\left(\varepsilon_{m}+\varepsilon_{n}-\varepsilon_{q}-\varepsilon_{r}\right)\left(\varepsilon_{m}-\varepsilon_{q}\right)} \\
& +\sum_{m>n}^{o c c} \sum_{q>r}^{\text {virt }} \frac{\left\langle n\left|\hat{h}_{S O}\right| r\right\rangle\left\langle m\left|\hat{h}_{S O}\right| q\right\rangle\left\langle q\left|\hat{h}_{S O}\right| m\right\rangle\left\langle r\left|\hat{h}_{S O}\right| n\right\rangle}{\left(\varepsilon_{n}-\varepsilon_{r}\right)\left(\varepsilon_{m}+\varepsilon_{n}-\varepsilon_{q}-\varepsilon_{r}\right)\left(\varepsilon_{n}-\varepsilon_{r}\right)} \\
& -\sum_{m>n}^{o c c} \sum_{q>r}^{\text {virt }} \frac{\left\langle n\left|\hat{h}_{S O}\right| r\right\rangle\left\langle m\left|\hat{h}_{S O}\right| q\right\rangle\left\langle r\left|\hat{h}_{S O}\right| m\right\rangle\left\langle q\left|\hat{h}_{S O}\right| n\right\rangle}{\left(\varepsilon_{n}-\varepsilon_{r}\right)\left(\varepsilon_{m}+\varepsilon_{n}-\varepsilon_{q}-\varepsilon_{r}\right)\left(\varepsilon_{n}-\varepsilon_{q}\right)} \\
& -\sum_{m>n}^{o c c} \sum_{q>r}^{v i r t} \frac{\left\langle n\left|\hat{h}_{S O}\right| r\right\rangle\left\langle m\left|\hat{h}_{S O}\right| q\right\rangle\left\langle q\left|\hat{h}_{S O}\right| n\right\rangle\left\langle r\left|\hat{h}_{S O}\right| m\right\rangle}{\left(\varepsilon_{n}-\varepsilon_{r}\right)\left(\varepsilon_{m}+\varepsilon_{n}-\varepsilon_{q}-\varepsilon_{r}\right)\left(\varepsilon_{m}-\varepsilon_{r}\right)} \\
& -\sum_{m>n}^{o c c} \sum_{q>r}^{v i r t} \frac{\left\langle n\left|\hat{h}_{S O}\right| q\right\rangle\left\langle m\left|\hat{h}_{S O}\right| r\right\rangle\left\langle r\left|\hat{h}_{S O}\right| n\right\rangle\left\langle q\left|\hat{h}_{S O}\right| m\right\rangle}{\left(\varepsilon_{n}-\varepsilon_{q}\right)\left(\varepsilon_{m}+\varepsilon_{n}-\varepsilon_{q}-\varepsilon_{r}\right)\left(\varepsilon_{m}-\varepsilon_{q}\right)} \\
& -\sum_{m>n} \sum_{q>r}^{\text {virt }} \frac{\left\langle n\left|\hat{h}_{S O}\right| q\right\rangle\left\langle m\left|\hat{h}_{S O}\right| r\right\rangle\left\langle q\left|\hat{h}_{S O}\right| m\right\rangle\left\langle r\left|\hat{h}_{S O}\right| n\right\rangle}{\left(\varepsilon_{n}-\varepsilon_{q}\right)\left(\varepsilon_{m}+\varepsilon_{n}-\varepsilon_{q}-\varepsilon_{r}\right)\left(\varepsilon_{n}-\varepsilon_{r}\right)} \\
& +\sum_{m>n}^{o c c} \sum_{q>r}^{v i r t} \frac{\left\langle n\left|\hat{h}_{S O}\right| q\right\rangle\left\langle m\left|\hat{h}_{S O}\right| r\right\rangle\left\langle r\left|\hat{h}_{S O}\right| m\right\rangle\left\langle q\left|\hat{h}_{S O}\right| n\right\rangle}{\left(\varepsilon_{n}-\varepsilon_{q}\right)\left(\varepsilon_{m}+\varepsilon_{n}-\varepsilon_{q}-\varepsilon_{r}\right)\left(\varepsilon_{n}-\varepsilon_{q}\right)} \\
& +\sum_{m>n}^{o c c} \sum_{q>r}^{v i r t} \frac{\left\langle n\left|\hat{h}_{S O}\right| q\right\rangle\left\langle m\left|\hat{h}_{S O}\right| r\right\rangle\left\langle q\left|\hat{h}_{S O}\right| n\right\rangle\left\langle r\left|\hat{h}_{S O}\right| m\right\rangle}{\left(\varepsilon_{n}-\varepsilon_{q}\right)\left(\varepsilon_{m}+\varepsilon_{n}-\varepsilon_{q}-\varepsilon_{r}\right)\left(\varepsilon_{m}-\varepsilon_{r}\right)} \\
& -\sum_{m>n}^{o c c} \sum_{q>r}^{v i r} \frac{\left\langle m\left|\hat{h}_{S O}\right| r\right\rangle\left\langle n\left|\hat{h}_{S O}\right| q\right\rangle\left\langle r\left|\hat{h}_{S O}\right| n\right\rangle\left\langle q\left|\hat{h}_{S O}\right| m\right\rangle}{\left(\varepsilon_{m}-\varepsilon_{r}\right)\left(\varepsilon_{m}+\varepsilon_{n}-\varepsilon_{q}-\varepsilon_{r}\right)\left(\varepsilon_{m}-\varepsilon_{q}\right)} \\
& -\sum_{m>n}^{o c c} \sum_{q>r}^{v i r} \frac{\left\langle m\left|\hat{h}_{S O}\right| r\right\rangle\left\langle n\left|\hat{h}_{S O}\right| q\right\rangle\left\langle r\left|\hat{h}_{S O}\right| m\right\rangle\left\langle q\left|\hat{h}_{S O}\right| n\right\rangle}{\left(\varepsilon_{m}-\varepsilon_{r}\right)\left(\varepsilon_{m}+\varepsilon_{n}-\varepsilon_{q}-\varepsilon_{r}\right)\left(\varepsilon_{n}-\varepsilon_{q}\right)} \\
& +\sum_{m>n}^{o c c} \sum_{q>r}^{\text {virt }} \frac{\left\langle m\left|\hat{h}_{S O}\right| r\right\rangle\left\langle n\left|\hat{h}_{S O}\right| q\right\rangle\left\langle r\left|\hat{h}_{S O}\right| m\right\rangle\left\langle q\left|\hat{h}_{S O}\right| n\right\rangle}{\left(\varepsilon_{m}-\varepsilon_{r}\right)\left(\varepsilon_{m}+\varepsilon_{n}-\varepsilon_{q}-\varepsilon_{r}\right)\left(\varepsilon_{n}-\varepsilon_{q}\right)} \\
& +\sum_{m>n}^{o c c} \sum_{q>r}^{\text {virt }} \frac{\left\langle m\left|\hat{h}_{S O}\right| r\right\rangle\left\langle n\left|\hat{h}_{S O}\right| q\right\rangle\left\langle q\left|\hat{h}_{S O}\right| n\right\rangle\left\langle r\left|\hat{h}_{S O}\right| m\right\rangle}{\left(\varepsilon_{m}-\varepsilon_{r}\right)\left(\varepsilon_{m}+\varepsilon_{n}-\varepsilon_{q}-\varepsilon_{r}\right)\left(\varepsilon_{m}-\varepsilon_{r}\right)} .
\end{aligned}
$$


Combining i) lines 1, 2, 5, 6 ii) lines 3, 4, 7, 8 iii) lines 9, 10, 13, 14 iv) lines 11, 12 ,15, 16 of Eq. (S11) and noticing that the sums can be extended to all values because the contributions cancel each other for the cases $m=n$ and $q=r$, we obtain:

$$
\begin{aligned}
& E_{D}^{(4,0)}=\frac{1}{4} \sum_{m, n}^{o c c} \sum_{q, r}^{\text {virt }} \frac{\left\langle m\left|\hat{h}_{S O}\right| q\right\rangle\left\langle n\left|\hat{h}_{S O}\right| r\right\rangle\left\langle r\left|\hat{h}_{S O}\right| n\right\rangle\left\langle q\left|\hat{h}_{S O}\right| m\right\rangle}{\left(\varepsilon_{m}+\varepsilon_{n}-\varepsilon_{q}-\varepsilon_{r}\right)} \\
& \times\left[\frac{1}{\left(\varepsilon_{m}-\varepsilon_{q}\right)\left(\varepsilon_{m}-\varepsilon_{q}\right)}+\frac{1}{\left(\varepsilon_{m}-\varepsilon_{q}\right)\left(\varepsilon_{n}-\varepsilon_{r}\right)}+\frac{1}{\left(\varepsilon_{n}-\varepsilon_{r}\right)\left(\varepsilon_{m}-\varepsilon_{q}\right)}+\frac{1}{\left(\varepsilon_{n}-\varepsilon_{r}\right)\left(\varepsilon_{n}-\varepsilon_{r}\right)}\right] \\
& -\frac{1}{4} \sum_{m, n}^{o c c} \sum_{q, r}^{v i r t} \frac{\left\langle m\left|\hat{h}_{S O}\right| q\right\rangle\left\langle n\left|\hat{h}_{S O}\right| r\right\rangle\left\langle r\left|\hat{h}_{S O}\right| m\right\rangle\left\langle q\left|\hat{h}_{S O}\right| n\right\rangle}{\left(\varepsilon_{m}+\varepsilon_{n}-\varepsilon_{q}-\varepsilon_{r}\right)} \\
& \times\left[\frac{1}{\left(\varepsilon_{m}-\varepsilon_{q}\right)\left(\varepsilon_{n}-\varepsilon_{q}\right)}+\frac{1}{\left(\varepsilon_{m}-\varepsilon_{q}\right)\left(\varepsilon_{m}-\varepsilon_{r}\right)}+\frac{1}{\left(\varepsilon_{n}-\varepsilon_{r}\right)\left(\varepsilon_{n}-\varepsilon_{q}\right)}+\frac{1}{\left(\varepsilon_{n}-\varepsilon_{r}\right)\left(\varepsilon_{m}-\varepsilon_{r}\right)}\right] \\
& -\frac{1}{4} \sum_{m, n}^{o c c} \sum_{q, r}^{\text {virt }} \frac{\left\langle n\left|\hat{h}_{S O}\right| q\right\rangle\left\langle m\left|\hat{h}_{S O}\right| r\right\rangle\left\langle r\left|\hat{h}_{S O}\right| n\right\rangle\left\langle q\left|\hat{h}_{S O}\right| m\right\rangle}{\left(\varepsilon_{m}+\varepsilon_{n}-\varepsilon_{q}-\varepsilon_{r}\right)} \\
& \times\left[\frac{1}{\left(\varepsilon_{n}-\varepsilon_{q}\right)\left(\varepsilon_{m}-\varepsilon_{q}\right)}+\frac{1}{\left(\varepsilon_{n}-\varepsilon_{q}\right)\left(\varepsilon_{n}-\varepsilon_{r}\right)}+\frac{1}{\left(\varepsilon_{m}-\varepsilon_{r}\right)\left(\varepsilon_{m}-\varepsilon_{q}\right)}+\frac{1}{\left(\varepsilon_{m}-\varepsilon_{r}\right)\left(\varepsilon_{n}-\varepsilon_{q}\right)}\right] \\
& +\frac{1}{4} \sum_{m, n}^{o c c} \sum_{q, r}^{\text {virt }} \frac{\left\langle n\left|\hat{h}_{S O}\right| q\right\rangle\left\langle m\left|\hat{h}_{S O}\right| r\right\rangle\left\langle r\left|\hat{h}_{S O}\right| m\right\rangle\left\langle q\left|\hat{h}_{S O}\right| n\right\rangle}{\left(\varepsilon_{m}+\varepsilon_{n}-\varepsilon_{q}-\varepsilon_{r}\right)} \\
& \times\left[\frac{1}{\left(\varepsilon_{n}-\varepsilon_{q}\right)\left(\varepsilon_{n}-\varepsilon_{q}\right)}+\frac{1}{\left(\varepsilon_{n}-\varepsilon_{q}\right)\left(\varepsilon_{m}-\varepsilon_{r}\right)}+\frac{1}{\left(\varepsilon_{m}-\varepsilon_{r}\right)\left(\varepsilon_{n}-\varepsilon_{q}\right)}+\frac{1}{\left(\varepsilon_{m}-\varepsilon_{r}\right)\left(\varepsilon_{m}-\varepsilon_{r}\right)}\right] .
\end{aligned}
$$

Then, combining the first and second terms, as well as the third and fourth terms of lines 2, 4, 6 and 8 of Eq. (S12) yields:

$$
\begin{aligned}
E_{D}^{(4,0)} & =\frac{1}{4} \sum_{m, n}^{o c c} \sum_{q, r}^{\text {virt }} \frac{\left\langle m\left|\hat{h}_{S O}\right| q\right\rangle\left\langle n\left|\hat{h}_{S O}\right| r\right\rangle\left\langle r\left|\hat{h}_{S O}\right| n\right\rangle\left\langle q\left|\hat{h}_{S O}\right| m\right\rangle}{\left(\varepsilon_{m}-\varepsilon_{q}\right)\left(\varepsilon_{m}-\varepsilon_{q}\right)\left(\varepsilon_{n}-\varepsilon_{r}\right)} \\
& +\frac{1}{4} \sum_{m, n}^{o c c} \sum_{q, r}^{\text {virt }} \frac{\left\langle m\left|\hat{h}_{S O}\right| q\right\rangle\left\langle n\left|\hat{h}_{S O}\right| r\right\rangle\left\langle r\left|\hat{h}_{S O}\right| n\right\rangle\left\langle q\left|\hat{h}_{S O}\right| m\right\rangle}{\left(\varepsilon_{m}-\varepsilon_{q}\right)\left(\varepsilon_{n}-\varepsilon_{r}\right)\left(\varepsilon_{n}-\varepsilon_{r}\right)} \\
& -\frac{1}{4} \sum_{m, n}^{o c c} \sum_{q, r}^{\text {virt }} \frac{\left\langle m\left|\hat{h}_{S O}\right| q\right\rangle\left\langle n\left|\hat{h}_{S O}\right| r\right\rangle\left\langle r\left|\hat{h}_{S O}\right| m\right\rangle\left\langle q\left|\hat{h}_{S O}\right| n\right\rangle}{\left(\varepsilon_{m}-\varepsilon_{q}\right)\left(\varepsilon_{n}-\varepsilon_{q}\right)\left(\varepsilon_{m}-\varepsilon_{r}\right)} \\
& -\frac{1}{4} \sum_{m, n}^{o c c} \sum_{q, r}^{v i r t} \frac{\left\langle m\left|\hat{h}_{S O}\right| q\right\rangle\left\langle n\left|\hat{h}_{S O}\right| r\right\rangle\left\langle r\left|\hat{h}_{S O}\right| m\right\rangle\left\langle q\left|\hat{h}_{S O}\right| n\right\rangle}{\left(\varepsilon_{n}-\varepsilon_{q}\right)\left(\varepsilon_{m}-\varepsilon_{r}\right)\left(\varepsilon_{n}-\varepsilon_{r}\right)} \\
& -\frac{1}{4} \sum_{m, n}^{o c c} \sum_{q, r}^{\text {virt }} \frac{\left\langle n\left|\hat{h}_{S O}\right| q\right\rangle\left\langle m\left|\hat{h}_{S O}\right| r\right\rangle\left\langle r\left|\hat{h}_{S O}\right| n\right\rangle\left\langle q\left|\hat{h}_{S O}\right| m\right\rangle}{\left(\varepsilon_{m}-\varepsilon_{q}\right)\left(\varepsilon_{n}-\varepsilon_{q}\right)\left(\varepsilon_{n}-\varepsilon_{r}\right)} \\
& -\frac{1}{4} \sum_{m, n}^{o c c} \sum_{q, r}^{v i r t} \frac{\left\langle n\left|\hat{h}_{S O}\right| q\right\rangle\left\langle m\left|\hat{h}_{S O}\right| r\right\rangle\left\langle r\left|\hat{h}_{S O}\right| n\right\rangle\left\langle q\left|\hat{h}_{S O}\right| m\right\rangle}{\left(\varepsilon_{m}-\varepsilon_{q}\right)\left(\varepsilon_{n}-\varepsilon_{q}\right)\left(\varepsilon_{m}-\varepsilon_{r}\right)} \\
& +\frac{1}{4} \sum_{m, n}^{o c c} \sum_{q, r}^{v i r t} \frac{\left\langle n\left|\hat{h}_{S O}\right| q\right\rangle\left\langle m\left|\hat{h}_{S O}\right| r\right\rangle\left\langle r\left|\hat{h}_{S O}\right| m\right\rangle\left\langle q\left|\hat{h}_{S O}\right| n\right\rangle}{\left(\varepsilon_{n}-\varepsilon_{q}\right)\left(\varepsilon_{n}-\varepsilon_{q}\right)\left(\varepsilon_{m}-\varepsilon_{r}\right)} \\
& +\frac{1}{4} \sum_{m, n}^{o c c} \sum_{q, r}^{v i r t} \frac{\left\langle n\left|\hat{h}_{S O}\right| q\right\rangle\left\langle m\left|\hat{h}_{S O}\right| r\right\rangle\left\langle r\left|\hat{h}_{S O}\right| m\right\rangle\left\langle q\left|\hat{h}_{S O}\right| n\right\rangle}{\left(\varepsilon_{n}-\varepsilon_{q}\right)\left(\varepsilon_{m}-\varepsilon_{r}\right)\left(\varepsilon_{m}-\varepsilon_{r}\right)} .
\end{aligned}
$$


A switch of the indices $n \leftrightarrow m$ in i) line 7 and then combining it with line 1 ii) line 8 and then combining it with line 2 iii) line 5 and then combining it with line 3 iv) line 6 and then combining it with line 4 of Eq. (S13) gives:

$$
\begin{aligned}
E_{D}^{(4,0)} & =\frac{1}{2} \sum_{m, n}^{o c c} \sum_{q, r}^{\text {virt }} \frac{\left\langle m\left|\hat{h}_{S O}\right| q\right\rangle\left\langle n\left|\hat{h}_{S O}\right| r\right\rangle\left\langle r\left|\hat{h}_{S O}\right| n\right\rangle\left\langle q\left|\hat{h}_{S O}\right| m\right\rangle}{\left(\varepsilon_{m}-\varepsilon_{q}\right)\left(\varepsilon_{m}-\varepsilon_{q}\right)\left(\varepsilon_{n}-\varepsilon_{r}\right)} \\
& +\frac{1}{2} \sum_{m, n}^{o c c} \sum_{q, r}^{\text {virt }} \frac{\left\langle m\left|\hat{h}_{S O}\right| q\right\rangle\left\langle n\left|\hat{h}_{S O}\right| r\right\rangle\left\langle r\left|\hat{h}_{S O}\right| n\right\rangle\left\langle q\left|\hat{h}_{S O}\right| m\right\rangle}{\left(\varepsilon_{m}-\varepsilon_{q}\right)\left(\varepsilon_{n}-\varepsilon_{r}\right)\left(\varepsilon_{n}-\varepsilon_{r}\right)} \\
& -\frac{1}{2} \sum_{m, n}^{o c c} \sum_{q, r}^{\text {virt }} \frac{\left\langle m\left|\hat{h}_{S O}\right| q\right\rangle\left\langle n\left|\hat{h}_{S O}\right| r\right\rangle\left\langle r\left|\hat{h}_{S O}\right| m\right\rangle\left\langle q\left|\hat{h}_{S O}\right| n\right\rangle}{\left(\varepsilon_{m}-\varepsilon_{q}\right)\left(\varepsilon_{n}-\varepsilon_{q}\right)\left(\varepsilon_{m}-\varepsilon_{r}\right)} \\
& -\frac{1}{2} \sum_{m, n}^{o c c} \sum_{q, r}^{\text {virt }} \frac{\left\langle m\left|\hat{h}_{S O}\right| q\right\rangle\left\langle n\left|\hat{h}_{S O}\right| r\right\rangle\left\langle r\left|\hat{h}_{S O}\right| m\right\rangle\left\langle q\left|\hat{h}_{S O}\right| n\right\rangle}{\left(\varepsilon_{n}-\varepsilon_{q}\right)\left(\varepsilon_{m}-\varepsilon_{r}\right)\left(\varepsilon_{n}-\varepsilon_{r}\right)}
\end{aligned}
$$

Switching the indices $m, q \leftrightarrow n, r$ in lines 2 and 4 of Eq. (S14) and then combining them with lines 1 and 3, we obtain:

$$
\begin{aligned}
E_{D}^{(4,0)} & =\sum_{m, n}^{o c c} \sum_{q, r}^{\text {virt }} \frac{\left\langle m\left|\hat{h}_{S O}\right| q\right\rangle\left\langle n\left|\hat{h}_{S O}\right| r\right\rangle\left\langle r\left|\hat{h}_{S O}\right| n\right\rangle\left\langle q\left|\hat{h}_{S O}\right| m\right\rangle}{\left(\varepsilon_{m}-\varepsilon_{q}\right)\left(\varepsilon_{m}-\varepsilon_{q}\right)\left(\varepsilon_{n}-\varepsilon_{r}\right)} \\
& -\sum_{m, n}^{o c c} \sum_{q, r}^{\text {virt }} \frac{\left\langle m\left|\hat{h}_{S O}\right| q\right\rangle\left\langle n\left|\hat{h}_{S O}\right| r\right\rangle\left\langle r\left|\hat{h}_{S O}\right| m\right\rangle\left\langle q\left|\hat{h}_{S O}\right| n\right\rangle}{\left(\varepsilon_{m}-\varepsilon_{q}\right)\left(\varepsilon_{n}-\varepsilon_{q}\right)\left(\varepsilon_{m}-\varepsilon_{r}\right)}
\end{aligned}
$$

Finally, renaming the indices $q \rightarrow p$ and $r \rightarrow q$ in Eq. (S15) gives us the following expression:

$$
\begin{aligned}
E_{D}^{(4,0)} & =\sum_{m, n}^{o c c} \sum_{p, q}^{\text {virt }} \frac{\left\langle m\left|\hat{h}_{S O}\right| p\right\rangle\left\langle n\left|\hat{h}_{S O}\right| q\right\rangle\left\langle q\left|\hat{h}_{S O}\right| n\right\rangle\left\langle p\left|\hat{h}_{S O}\right| m\right\rangle}{\left(\varepsilon_{m}-\varepsilon_{p}\right)\left(\varepsilon_{n}-\varepsilon_{q}\right)\left(\varepsilon_{m}-\varepsilon_{p}\right)} \\
& -\sum_{m, n}^{o c c} \sum_{p, q}^{\text {virt }} \frac{\left\langle m\left|\hat{h}_{S O}\right| p\right\rangle\left\langle n\left|\hat{h}_{S O}\right| q\right\rangle\left\langle q\left|\hat{h}_{S O}\right| m\right\rangle\left\langle p\left|\hat{h}_{S O}\right| n\right\rangle}{\left(\varepsilon_{m}-\varepsilon_{p}\right)\left(\varepsilon_{n}-\varepsilon_{p}\right)\left(\varepsilon_{m}-\varepsilon_{q}\right)} .
\end{aligned}
$$

\section{Combining $E_{S}^{(4,0)}$ and $E_{D}^{(4,0)}$}

Taking the sum of Eqs. (S6) and (S16) gives:

$$
\begin{aligned}
E^{(4,0)} & =-\sum_{m, n}^{o c c} \sum_{p, q}^{\text {virt }} 2 R\left[\frac{\left\langle m\left|\hat{h}_{S O}\right| p\right\rangle\left\langle p\left|\hat{h}_{S O}\right| q\right\rangle\left\langle n\left|\hat{h}_{S O}\right| m\right\rangle\left\langle q\left|\hat{h}_{S O}\right| n\right\rangle}{\left(\varepsilon_{m}-\varepsilon_{p}\right)\left(\varepsilon_{n}-\varepsilon_{q}\right)\left(\varepsilon_{m}-\varepsilon_{q}\right)}\right] \\
& +\sum_{m}^{o c c} \sum_{p, q, r}^{\text {virt }} \frac{\left\langle m\left|\hat{h}_{S O}\right| p\right\rangle\left\langle p\left|\hat{h}_{S O}\right| q\right\rangle\left\langle q\left|\hat{h}_{S O}\right| r\right\rangle\left\langle r\left|\hat{h}_{S O}\right| m\right\rangle}{\left(\varepsilon_{m}-\varepsilon_{p}\right)\left(\varepsilon_{m}-\varepsilon_{q}\right)\left(\varepsilon_{m}-\varepsilon_{r}\right)} \\
& +\sum_{m, n, o}^{o c c} \sum_{p}^{\text {virt }} \frac{\left\langle m\left|\hat{h}_{S O}\right| p\right\rangle\left\langle n\left|\hat{h}_{S O}\right| m\right\rangle\left\langle o\left|\hat{h}_{S O}\right| n\right\rangle\left\langle p\left|\hat{h}_{S O}\right| o\right\rangle}{\left(\varepsilon_{m}-\varepsilon_{p}\right)\left(\varepsilon_{n}-\varepsilon_{p}\right)\left(\varepsilon_{o}-\varepsilon_{p}\right)} \\
& -\sum_{m, n}^{o c c} \sum_{p, q}^{\text {virt }} \frac{\left\langle m\left|\hat{h}_{S O}\right| p\right\rangle\left\langle n\left|\hat{h}_{S O}\right| q\right\rangle\left\langle q\left|\hat{h}_{S O}\right| m\right\rangle\left\langle p\left|\hat{h}_{S O}\right| n\right\rangle}{\left(\varepsilon_{m}-\varepsilon_{p}\right)\left(\varepsilon_{n}-\varepsilon_{p}\right)\left(\varepsilon_{m}-\varepsilon_{q}\right)} .
\end{aligned}
$$


Substituting Eqs. (15a) and (15b) into Eq. (S17), we obtain:

$$
\begin{aligned}
E^{(4,0)} & =\sum_{\sigma \sigma^{\prime} \sigma^{\prime \prime} \sigma^{\prime \prime}}\left[2 \sum_{m, n}^{o c c} \sum_{p, q}^{\text {virt }} \frac{U_{m p}^{\sigma \sigma^{\prime}(1,0)} G_{p q}^{\sigma^{\prime} \sigma^{\prime \prime}(1,0)} G_{n m}^{\sigma^{\prime \prime \prime} \sigma^{\prime}(1,0)} U_{q n}^{\sigma^{\prime \prime} \sigma^{\prime \prime \prime}(1,0)}}{\varepsilon_{m}^{\sigma}-\varepsilon_{q}^{\sigma^{\prime \prime}}}\right. \\
& -\sum_{m} \sum_{p, q, r}^{o c c} \frac{U_{m p}^{\sigma \sigma^{\prime}(1,0)} G_{p q}^{\sigma^{\prime} \sigma^{\prime \prime}(1,0)} G_{q r}^{\sigma^{\prime \prime} \sigma^{\prime \prime \prime}(1,0)} U_{r m}^{\sigma^{\prime \prime \prime} \sigma(1,0)}}{\varepsilon_{m}^{\sigma}-\varepsilon_{q}^{\sigma^{\prime \prime}}} \\
& -\sum_{m, n, o}^{o c c} \sum_{p}^{\text {virt }} \frac{U_{m p}^{\sigma \sigma^{\prime}(1,0)} G_{n m}^{\sigma^{\prime \prime} \sigma(1,0)} G_{o n}^{\sigma^{\prime \prime \prime} \sigma^{\prime \prime}(1,0)} U_{p o}^{\sigma^{\prime} \sigma^{\prime \prime \prime}(1,0)}}{\varepsilon_{n}^{\sigma^{\prime \prime}}-\varepsilon_{p}^{\sigma^{\prime}}} \\
& \left.+\sum_{m, n}^{o c c} \sum_{p, q}^{\text {virt }} U_{m p}^{\sigma \sigma^{\prime}(1,0)} U_{p n}^{\sigma^{\prime} \sigma^{\prime \prime}(1,0)} U_{q m}^{\sigma^{\prime \prime \prime} \sigma(1,0)} U_{n q}^{\sigma^{\prime \prime} \sigma^{\prime \prime \prime}(1,0)}\left(\varepsilon_{q}^{\sigma^{\prime \prime \prime}}-\varepsilon_{n}^{\sigma^{\prime \prime}}\right)\right] .
\end{aligned}
$$

\section{DETAILS ON THE DERIVATION OF EQUATIONS B19 AND B20}

\section{A. The Derivation of Equation B19 for $E_{S}^{(3,1)}$}

The expression for $E_{S}^{(3,1)}$ can be obtained from Eq. (21) of the main text, by limiting the sum over configurations to only singly-excited determinants:

$$
\begin{aligned}
E_{S}^{(3,1)} & =\sum_{I J K}^{S} 2 R\left[\left\langle\psi_{I}^{(0,0)}\left|\hat{H}^{(0,1)}-E^{(0,1)}\right| \psi_{J}^{(0,0)}\right\rangle \frac{\left\langle\psi^{(0,0)}\left|\hat{h}_{S O}\right| \psi_{I}^{(0,0)}\right\rangle\left\langle\psi_{J}^{(0,0)}\left|\hat{h}_{S O}\right| \psi_{K}^{(0,0)}\right\rangle\left\langle\psi_{K}^{(0,0)}\left|\hat{h}_{S O}\right| \psi^{(0,0)}\right\rangle}{\left(E^{(0,0)}-E_{I}^{(0,0)}\right)\left(E^{(0,0)}-E_{J}^{(0,0)}\right)\left(E^{(0,0)}-E_{K}^{(0,0)}\right)}\right] \\
& +\sum_{I J K}^{S} 2 R\left[\left\langle\psi^{(0,0)}\left|\hat{H}^{(0,1)}\right| \psi_{I}^{(0,0)}\right\rangle \frac{\left\langle\psi_{I}^{(0,0)}\left|\hat{h}_{S O}\right| \psi_{J}^{(0,0)}\right\rangle\left\langle\psi_{J}^{(0,0)}\left|\hat{h}_{S O}\right| \psi_{K}^{(0,0)}\right\rangle\left\langle\psi_{K}^{(0,0)}\left|\hat{h}_{S O}\right| \psi^{(0,0)}\right\rangle}{\left(E^{(0,0)}-E_{I}^{(0,0)}\right)\left(E^{(0,0)}-E_{J}^{(0,0)}\right)\left(E^{(0,0)}-E_{K}^{(0,0)}\right)}\right] .
\end{aligned}
$$

We can see immediately in Eq. (S19) that the sum in the second line vanishes by applying Brillouin's theorem to the matrix element $\left\langle\psi^{(0,0)}\left|\hat{H}^{(0,1)}\right| \psi_{I}^{(0,0)}\right\rangle$. Then, in the first line we begin by expressing $\left|\psi_{I}^{(0,0)}\right\rangle,\left|\psi_{J}^{(0,0)}\right\rangle$ and $\left|\psi_{K}^{(0,0)}\right\rangle$ as generic singlyexcited determinants $\left|\psi_{m}^{p}\right\rangle,\left|\psi_{n}^{q}\right\rangle$ and $\left|\psi_{o}^{r}\right\rangle$ :

$$
E_{S}^{(3,1)}=\sum_{m, n, o}^{o c c} \sum_{p, q, r}^{v i r t} 2 R\left[\left\langle\psi_{m}^{p}\left|\hat{V}_{e e}-(\hat{C}-\hat{K})-E^{(0,1)}\right| \psi_{n}^{q}\right\rangle \frac{\left\langle\psi^{(0,0)}\left|\hat{h}_{S O}\right| \psi_{m}^{p}\right\rangle\left\langle\psi_{n}^{q}\left|\hat{h}_{S O}\right| \psi_{o}^{r}\right\rangle\left\langle\psi_{o}^{r}\left|\hat{h}_{S O}\right| \psi^{(0,0)}\right\rangle}{\left(E^{(0,0)}-E_{m}^{p}\right)\left(E^{(0,0)}-E_{n}^{q}\right)\left(E^{(0,0)}-E_{o}^{r}\right)}\right] .
$$

We can now evaluate all integrals in Eq. (S20) using the Slater-Condon rules. For integrals involving singly-excited determinants and the electron fluctuation potential:

$$
\left\langle\psi_{m}^{p}\left|\hat{V}_{e e}-(\hat{C}-\hat{K})-E^{(0,1)}\right| \psi_{n}^{q}\right\rangle=(p m \mid n q)-(p q \mid n m)
$$

Substituting Eqs. (S3a)-(S3c) and (S21a) in Eq. (S20) gives:

$$
E_{S}^{(3,1)}=\sum_{m, n, o}^{o c c} \sum_{p, q, r}^{v i r t} 2 R\left[\{(p m \mid n q)-(p q \mid n m)\} \frac{\left\langle m\left|\hat{h}_{S O}\right| p\right\rangle\left\{\delta_{o, n}\left\langle q\left|\hat{h}_{S O}\right| r\right\rangle-\delta_{q, r}\left\langle o\left|\hat{h}_{S O}\right| n\right\rangle\right\}\left\langle r\left|\hat{h}_{S O}\right| o\right\rangle}{\left(\varepsilon_{m}-\varepsilon_{p}\right)\left(\varepsilon_{n}-\varepsilon_{q}\right)\left(\varepsilon_{o}-\varepsilon_{r}\right)}\right] .
$$

Evaluating the Kronecker deltas, we find an expression that is equivalent to Eq. (B19) of the main text:

$$
\begin{aligned}
E_{S}^{(3,1)} & =\sum_{m, n}^{o c c} \sum_{p, q, r}^{\text {virt }} 2 R\left[[(p m \mid n q)-(p q \mid n m)] \frac{\left\langle m\left|\hat{h}_{S O}\right| p\right\rangle\left\langle q\left|\hat{h}_{S O}\right| r\right\rangle\left\langle r\left|\hat{h}_{S O}\right| n\right\rangle}{\left(\varepsilon_{m}-\varepsilon_{p}\right)\left(\varepsilon_{n}-\varepsilon_{q}\right)\left(\varepsilon_{n}-\varepsilon_{r}\right)}\right] \\
& +\sum_{m, n, o}^{o c c} \sum_{p, q}^{v i r t} 2 R\left[[(p q \mid n m)-(p m \mid n q)] \frac{\left\langle m\left|\hat{h}_{S O}\right| p\right\rangle\left\langle o\left|\hat{h}_{S O}\right| n\right\rangle\left\langle q\left|\hat{h}_{S O}\right| o\right\rangle}{\left(\varepsilon_{m}-\varepsilon_{p}\right)\left(\varepsilon_{n}-\varepsilon_{q}\right)\left(\varepsilon_{o}-\varepsilon_{q}\right)}\right] .
\end{aligned}
$$




\section{B. The Derivation of Equation $\mathbf{B 2 0}$ for $E_{D}^{(3,1)}$}

We now consider the contribution $E_{D}^{(3,1)}$ from Eq.(21), which arises from doubly-excited (as well as singly-excited) configurations

$$
\begin{aligned}
E_{D}^{(3,1)} & =\sum_{I J K}^{S D} 2 R\left[\left\langle\psi_{I}^{(0,0)}\left|\hat{H}^{(0,1)}-E^{(0,1)}\right| \psi_{J}^{(0,0)}\right\rangle \frac{\left\langle\psi^{(0,0)}\left|\hat{h}_{S O}\right| \psi_{I}^{(0,0)}\right\rangle\left\langle\psi_{J}^{(0,0)}\left|\hat{h}_{S O}\right| \psi_{K}^{(0,0)}\right\rangle\left\langle\psi_{K}^{(0,0)}\left|\hat{h}_{S O}\right| \psi^{(0,0)}\right\rangle}{\left(E^{(0,0)}-E_{I}^{(0,0)}\right)\left(E^{(0,0)}-E_{J}^{(0,0)}\right)\left(E^{(0,0)}-E_{K}^{(0,0)}\right)}\right] \\
& +\sum_{I J K}^{S D} 2 R\left[\left\langle\psi^{(0,0)}\left|\hat{H}^{(0,1)}\right| \psi_{I}^{(0,0)}\right\rangle \frac{\left\langle\psi_{I}^{(0,0)}\left|\hat{h}_{S O}\right| \psi_{J}^{(0,0)}\right\rangle\left\langle\psi_{J}^{(0,0)}\left|\hat{h}_{S O}\right| \psi_{K}^{(0,0)}\right\rangle\left\langle\psi_{K}^{(0,0)}\left|\hat{h}_{S O}\right| \psi^{(0,0)}\right\rangle}{\left(E^{(0,0)}-E_{I}^{(0,0)}\right)\left(E^{(0,0)}-E_{J}^{(0,0)}\right)\left(E^{(0,0)}-E_{K}^{(0,0)}\right)}\right]
\end{aligned}
$$

Here the notation $S D$ on the summations indicates that at least one of the indices $I, J$ and $K$ in the sums must correspond to a doubly-excited configuration. For the first line of Eq. (S24), given that the matrix-elements $\left\langle\psi^{(0,0)}\left|\hat{h}_{S O}\right| \psi_{I}^{(0,0)}\right\rangle$ and $\left\langle\psi_{K}^{(0,0)}\left|\hat{h}_{S O}\right| \psi^{(0,0)}\right\rangle$ are present, the indices $I$ and $K$ can only be singly-excited configurations. This means that $J$ must be a doublyexcited configuration. In the second line of Eq. (S24) on the other hand, the presence of the matrix element $\left\langle\psi^{(0,0)}\left|\hat{H}^{(0,1)}\right| \psi_{I}^{(0,0)}\right\rangle$ shows us that $\left|\psi_{I}^{(0,0)}\right\rangle$ must be a doubly excited configuration. Although $K$ must be singly-excited, $J$ may be either:

$$
\begin{aligned}
E_{D}^{(3,1)} & =\sum_{I K}^{S} \sum_{J}^{D} 2 R\left[\left\langle\psi_{I}^{(0,0)}\left|\hat{H}^{(0,1)}-E^{(0,1)}\right| \psi_{J}^{(0,0)}\right\rangle \frac{\left\langle\psi^{(0,0)}\left|\hat{h}_{S O}\right| \psi_{I}^{(0,0)}\right\rangle\left\langle\psi_{J}^{(0,0)}\left|\hat{h}_{S O}\right| \psi_{K}^{(0,0)}\right\rangle\left\langle\psi_{K}^{(0,0)}\left|\hat{h}_{S O}\right| \psi^{(0,0)}\right\rangle}{\left(E^{(0,0)}-E_{I}^{(0,0)}\right)\left(E^{(0,0)}-E_{J}^{(0,0)}\right)\left(E^{(0,0)}-E_{K}^{(0,0)}\right)}\right] \\
& +\sum_{K}^{S} \sum_{J}^{\prime} \sum_{I}^{D} 2 R\left[\left\langle\psi^{(0,0)}\left|\hat{H}^{(0,1)}\right| \psi_{I}^{(0,0)}\right\rangle \frac{\left\langle\psi_{I}^{(0,0)}\left|\hat{h}_{S O}\right| \psi_{J}^{(0,0)}\right\rangle\left\langle\psi_{J}^{(0,0)}\left|\hat{h}_{S O}\right| \psi_{K}^{(0,0)}\right\rangle\left\langle\psi_{K}^{(0,0)}\left|\hat{h}_{S O}\right| \psi^{(0,0)}\right\rangle}{\left(E^{(0,0)}-E_{I}^{(0,0)}\right)\left(E^{(0,0)}-E_{J}^{(0,0)}\right)\left(E^{(0,0)}-E_{K}^{(0,0)}\right)}\right] \\
& =E_{D 1}^{(3,1)}+E_{D 2}^{(3,1)}
\end{aligned}
$$

where $E_{D 1}^{(3,1)}$ corresponds to the first line of Eq. (S25) and $E_{D 2}^{(3,1)}$ corresponds to the second line.

Writing first $E_{D 1}^{(3,1)}$ in terms of the corresponding generic singly- and doubly-excited configurations:

$$
E_{D 1}^{(3,1)}=\sum_{l, o}^{o c c} \sum_{m>n}^{o c c} \sum_{p, s}^{\text {virt }} \sum_{q>r}^{v i r t} 2 R\left[\left\langle\psi_{l}^{p}\left|\hat{H}^{(0,1)}-E^{(0,1)}\right| \psi_{m n}^{q r}\right\rangle \frac{\left\langle\psi^{(0,0)}\left|\hat{h}_{S O}\right| \psi_{l}^{p}\right\rangle\left\langle\psi_{m n}^{q r}\left|\hat{h}_{S O}\right| \psi_{o}^{s}\right\rangle\left\langle\psi_{o}^{s}\left|\hat{h}_{S O}\right| \psi^{(0,0)}\right\rangle}{\left(E^{(0,0)}-E_{l}^{p}\right)\left(E^{(0,0)}-E_{m n}^{q r}\right)\left(E^{(0,0)}-E_{o}^{s}\right)}\right]
$$

and for $E_{D 2}^{(3,1)}$, we have the following by expressing the second line of Eq. (S25) in terms of generic singly and doubly-excited configurations:

$$
\begin{aligned}
E_{D 2}^{(3,1)} & =\sum_{l, o}^{o c c} \sum_{m>n}^{o c c} \sum_{p, s}^{\text {virt }} \sum_{q>r}^{\text {virt }} 2 R\left[\left\langle\psi^{(0,0)}\left|\hat{H}^{(0,1)}\right| \psi_{m n}^{q r}\right\rangle \frac{\left\langle\psi_{m n}^{q r}\left|\hat{h}_{S O}\right| \psi_{l}^{p}\right\rangle\left\langle\psi_{l}^{p}\left|\hat{h}_{S O}\right| \psi_{o}^{s}\right\rangle\left\langle\psi_{o}^{s}\left|\hat{h}_{S O}\right| \psi^{(0,0)}\right\rangle}{\left(E^{(0,0)}-E_{m n}^{q r}\right)\left(E^{(0,0)}-E_{l}^{p}\right)\left(E^{(0,0)}-E_{o}^{s}\right)}\right] \\
& +\sum_{k}^{o c c} \sum_{l>m}^{o c c} \sum_{n, o}^{o c c} \sum_{p}^{\text {virt }} \sum_{q>r}^{\text {virt }} \sum_{s, t}^{\text {virt }} 2 R\left[\left\langle\psi^{(0,0)}\left|\hat{H}^{(0,1)}\right| \psi_{l m}^{q r}\right\rangle \frac{\left\langle\psi_{l m}^{q r}\left|\hat{h}_{S O}\right| \psi_{n o}^{s t}\right\rangle\left\langle\psi_{n o}^{s t}\left|\hat{h}_{S O}\right| \psi_{k}^{p}\right\rangle\left\langle\psi_{k}^{p}\left|\hat{h}_{S O}\right| \psi^{(0,0)}\right\rangle}{\left(E^{(0,0)}-E_{l m}^{q r}\right)\left(E^{(0,0)}-E_{n o}^{s t}\right)\left(E^{(0,0)}-E_{k}^{p}\right)}\right]
\end{aligned}
$$

\section{The Contribution $E_{D 1}^{(3,1)}$}

We can evaluate the integral in Eq. (S26) using the following formula for mixed electron repulsion integrals involving both singly- and doubly-excited determinants:

$$
\begin{aligned}
\left\langle\psi_{l}^{p}\left|\hat{V}_{e e}-(\hat{C}-\hat{K})-E^{(0,1)}\right| \psi_{m n}^{q r}\right\rangle & =\delta_{l, m}[(p q \mid n r)-(p r \mid n q)]+\delta_{l, n}[(m q \mid p r)-(m r \mid p q)] \\
& +\delta_{p, q}[(m r \mid n l)-(m l \mid n r)]+\delta_{p, r}[(m l \mid n q)-(m q \mid n l)] .
\end{aligned}
$$

Substituting Eq. (S9b) and Eq. (S28) in Eq. (S26), we get:

$$
\begin{aligned}
E_{D 1}^{(3,1)} & =\sum_{l, o}^{o c c} \sum_{m>n}^{o c c} \sum_{p, s}^{\text {virt }} \sum_{q>r}^{\text {virt }} 2 R\left[\left\{\delta_{l, m}[(p q \mid n r)-(p r \mid n q)]+\delta_{l, n}[(m q \mid p r)-(m r \mid p q)]+\delta_{p, q}[(m r \mid n l)-(m l \mid n r)]+\delta_{p, r}[(m l \mid n q)-(m q \mid n l)]\right\}\right. \\
& \left.\times \frac{\left\langle l\left|\hat{h}_{S O}\right| p\right\rangle\left\{\delta_{s, q} \delta_{o, m}\left\langle r\left|\hat{h}_{S O}\right| n\right\rangle+\delta_{s, r} \delta_{o, n}\left\langle q\left|\hat{h}_{S O}\right| m\right\rangle-\delta_{s, q} \delta_{o, n}\left\langle r\left|\hat{h}_{S O}\right| m\right\rangle-\delta_{s, r} \delta_{o, m}\left\langle q\left|\hat{h}_{S O}\right| n\right\rangle\right\}\left\langle s\left|\hat{h}_{S O}\right| o\right\rangle}{\left(\varepsilon_{l}-\varepsilon_{p}\right)\left(\varepsilon_{m}+\varepsilon_{n}-\varepsilon_{q}-\varepsilon_{r}\right)\left(\varepsilon_{o}-\varepsilon_{S}\right)}\right] .
\end{aligned}
$$


Evaluating the Kronecker deltas in Eq. (S29) gives:

$$
\begin{aligned}
& E_{D 1}^{(3,1)}=\sum_{m>n}^{o c c} \sum_{p}^{\text {virt }} \sum_{q>r}^{\text {virt }} 2 R\left[\{(p q \mid n r)-(p r \mid n q)\} \frac{\left\langle m\left|\hat{h}_{S O}\right| p\right\rangle\left\langle r\left|\hat{h}_{S O}\right| n\right\rangle\left\langle q\left|\hat{h}_{S O}\right| m\right\rangle}{\left(\varepsilon_{m}-\varepsilon_{p}\right)\left(\varepsilon_{m}+\varepsilon_{n}-\varepsilon_{q}-\varepsilon_{r}\right)\left(\varepsilon_{m}-\varepsilon_{q}\right)}\right] \\
& +\sum_{m>n}^{o c c} \sum_{p}^{\text {virt }} \sum_{q>r}^{v i r t} 2 R\left[\{(p q \mid n r)-(p r \mid n q)\} \frac{\left\langle m\left|\hat{h}_{S O}\right| p\right\rangle\left\langle q\left|\hat{h}_{S O}\right| m\right\rangle\left\langle r\left|\hat{h}_{S O}\right| n\right\rangle}{\left(\varepsilon_{m}-\varepsilon_{p}\right)\left(\varepsilon_{m}+\varepsilon_{n}-\varepsilon_{q}-\varepsilon_{r}\right)\left(\varepsilon_{n}-\varepsilon_{r}\right)}\right] \\
& -\sum_{m>n}^{o c c} \sum_{p}^{\text {virt }} \sum_{q>r}^{\text {virt }} 2 R\left[\{(p q \mid n r)-(p r \mid n q)\} \frac{\left\langle m\left|\hat{h}_{S O}\right| p\right\rangle\left\langle r\left|\hat{h}_{S O}\right| m\right\rangle\left\langle q\left|\hat{h}_{S O}\right| n\right\rangle}{\left(\varepsilon_{m}-\varepsilon_{p}\right)\left(\varepsilon_{m}+\varepsilon_{n}-\varepsilon_{q}-\varepsilon_{r}\right)\left(\varepsilon_{n}-\varepsilon_{q}\right)}\right] \\
& -\sum_{m>n}^{o c c} \sum_{p}^{v i r t} \sum_{q>r}^{v i r t} 2 R\left[\{(p q \mid n r)-(p r \mid n q)\} \frac{\left\langle m\left|\hat{h}_{S O}\right| p\right\rangle\left\langle q\left|\hat{h}_{S O}\right| n\right\rangle\left\langle r\left|\hat{h}_{S O}\right| m\right\rangle}{\left(\varepsilon_{m}-\varepsilon_{p}\right)\left(\varepsilon_{m}+\varepsilon_{n}-\varepsilon_{q}-\varepsilon_{r}\right)\left(\varepsilon_{m}-\varepsilon_{r}\right)}\right] \\
& +\sum_{m>n}^{o c c} \sum_{p}^{v i r t} \sum_{q>r}^{v i r t} 2 R\left[\{(m q \mid p r)-(m r \mid p q)\} \frac{\left\langle n\left|\hat{h}_{S O}\right| p\right\rangle\left\langle r\left|\hat{h}_{S O}\right| n\right\rangle\left\langle q\left|\hat{h}_{S O}\right| m\right\rangle}{\left(\varepsilon_{n}-\varepsilon_{p}\right)\left(\varepsilon_{m}+\varepsilon_{n}-\varepsilon_{q}-\varepsilon_{r}\right)\left(\varepsilon_{m}-\varepsilon_{q}\right)}\right] \\
& +\sum_{m>n}^{o c c} \sum_{p}^{\text {virt }} \sum_{q>r}^{v i r t} 2 R\left[\{(m q \mid p r)-(m r \mid p q)\} \frac{\left\langle n\left|\hat{h}_{S O}\right| p\right\rangle\left\langle q\left|\hat{h}_{S O}\right| m\right\rangle\left\langle r\left|\hat{h}_{S O}\right| n\right\rangle}{\left(\varepsilon_{n}-\varepsilon_{p}\right)\left(\varepsilon_{m}+\varepsilon_{n}-\varepsilon_{q}-\varepsilon_{r}\right)\left(\varepsilon_{n}-\varepsilon_{r}\right)}\right] \\
& -\sum_{m>n}^{o c c} \sum_{p}^{\text {virt }} \sum_{q>r}^{\text {virt }} 2 R\left[\{(m q \mid p r)-(m r \mid p q)\} \frac{\left\langle n\left|\hat{h}_{S O}\right| p\right\rangle\left\langle r\left|\hat{h}_{S O}\right| m\right\rangle\left\langle q\left|\hat{h}_{S O}\right| n\right\rangle}{\left(\varepsilon_{n}-\varepsilon_{p}\right)\left(\varepsilon_{m}+\varepsilon_{n}-\varepsilon_{q}-\varepsilon_{r}\right)\left(\varepsilon_{n}-\varepsilon_{q}\right)}\right] \\
& -\sum_{m>n}^{o c c} \sum_{p}^{\text {virt }} \sum_{q>r}^{\text {virt }} 2 R\left[\{(m q \mid p r)-(m r \mid p q)\} \frac{\left\langle n\left|\hat{h}_{S O}\right| p\right\rangle\left\langle q\left|\hat{h}_{S O}\right| n\right\rangle\left\langle r\left|\hat{h}_{S O}\right| m\right\rangle}{\left(\varepsilon_{n}-\varepsilon_{p}\right)\left(\varepsilon_{m}+\varepsilon_{n}-\varepsilon_{q}-\varepsilon_{r}\right)\left(\varepsilon_{m}-\varepsilon_{r}\right)}\right] \\
& +\sum_{l}^{o c c} \sum_{m>n}^{o c c} \sum_{q>r}^{v i r t} 2 R\left[\{(m r \mid n l)-(m l \mid n r)\} \frac{\left\langle l\left|\hat{h}_{S O}\right| q\right\rangle\left\langle r\left|\hat{h}_{S O}\right| n\right\rangle\left\langle q\left|\hat{h}_{S O}\right| m\right\rangle}{\left(\varepsilon_{l}-\varepsilon_{q}\right)\left(\varepsilon_{m}+\varepsilon_{n}-\varepsilon_{q}-\varepsilon_{r}\right)\left(\varepsilon_{m}-\varepsilon_{q}\right)}\right] \\
& +\sum_{l}^{o c c} \sum_{m>n}^{o c c} \sum_{q>r}^{\text {virt }} 2 R\left[\{(m r \mid n l)-(m l \mid n r)\} \frac{\left\langle l\left|\hat{h}_{S O}\right| q\right\rangle\left\langle q\left|\hat{h}_{S O}\right| m\right\rangle\left\langle r\left|\hat{h}_{S O}\right| n\right\rangle}{\left(\varepsilon_{l}-\varepsilon_{q}\right)\left(\varepsilon_{m}+\varepsilon_{n}-\varepsilon_{q}-\varepsilon_{r}\right)\left(\varepsilon_{n}-\varepsilon_{r}\right)}\right] \\
& -\sum_{l}^{o c c} \sum_{m>n}^{o c c} \sum_{q>r}^{v i r t} 2 R\left[\{(m r \mid n l)-(m l \mid n r)\} \frac{\left\langle l\left|\hat{h}_{S O}\right| q\right\rangle\left\langle r\left|\hat{h}_{S O}\right| m\right\rangle\left\langle q\left|\hat{h}_{S O}\right| n\right\rangle}{\left(\varepsilon_{l}-\varepsilon_{q}\right)\left(\varepsilon_{m}+\varepsilon_{n}-\varepsilon_{q}-\varepsilon_{r}\right)\left(\varepsilon_{n}-\varepsilon_{q}\right)}\right] \\
& -\sum_{l}^{o c c} \sum_{m>n}^{o c c} \sum_{q>r}^{v i r t} 2 R\left[\{(m r \mid n l)-(m l \mid n r)\} \frac{\left\langle l\left|\hat{h}_{S O}\right| q\right\rangle\left\langle q\left|\hat{h}_{S O}\right| n\right\rangle\left\langle r\left|\hat{h}_{S O}\right| m\right\rangle}{\left(\varepsilon_{l}-\varepsilon_{q}\right)\left(\varepsilon_{m}+\varepsilon_{n}-\varepsilon_{q}-\varepsilon_{r}\right)\left(\varepsilon_{m}-\varepsilon_{r}\right)}\right] \\
& +\sum_{l}^{o c c} \sum_{m>n}^{o c c} \sum_{q>r}^{v i r t} 2 R\left[\{(m l \mid n q)-(m q \mid n l)\} \frac{\left\langle l\left|\hat{h}_{S O}\right| r\right\rangle\left\langle r\left|\hat{h}_{S O}\right| n\right\rangle\left\langle q\left|\hat{h}_{S O}\right| m\right\rangle}{\left(\varepsilon_{l}-\varepsilon_{r}\right)\left(\varepsilon_{m}+\varepsilon_{n}-\varepsilon_{q}-\varepsilon_{r}\right)\left(\varepsilon_{m}-\varepsilon_{q}\right)}\right] \\
& +\sum_{l}^{o c c} \sum_{m>n}^{o c c} \sum_{q>r}^{v i r t} 2 R\left[\{(m l \mid n q)-(m q \mid n l)\} \frac{\left\langle l\left|\hat{h}_{S O}\right| r\right\rangle\left\langle q\left|\hat{h}_{S O}\right| m\right\rangle\left\langle r\left|\hat{h}_{S O}\right| n\right\rangle}{\left(\varepsilon_{l}-\varepsilon_{r}\right)\left(\varepsilon_{m}+\varepsilon_{n}-\varepsilon_{q}-\varepsilon_{r}\right)\left(\varepsilon_{n}-\varepsilon_{r}\right)}\right] \\
& -\sum_{l}^{o c c} \sum_{m>n}^{o c c} \sum_{q>r}^{v i r t} 2 R\left[\{(m l \mid n q)-(m q \mid n l)\} \frac{\left\langle l\left|\hat{h}_{S O}\right| r\right\rangle\left\langle r\left|\hat{h}_{S O}\right| m\right\rangle\left\langle q\left|\hat{h}_{S O}\right| n\right\rangle}{\left(\varepsilon_{l}-\varepsilon_{r}\right)\left(\varepsilon_{m}+\varepsilon_{n}-\varepsilon_{q}-\varepsilon_{r}\right)\left(\varepsilon_{n}-\varepsilon_{q}\right)}\right] \\
& -\sum_{l}^{o c c} \sum_{m>n}^{o c c} \sum_{q>r}^{v i r t} 2 R\left[\{(m l \mid n q)-(m q \mid n l)\} \frac{\left\langle l\left|\hat{h}_{S O}\right| r\right\rangle\left\langle q\left|\hat{h}_{S O}\right| n\right\rangle\left\langle r\left|\hat{h}_{S O}\right| m\right\rangle}{\left(\varepsilon_{l}-\varepsilon_{r}\right)\left(\varepsilon_{m}+\varepsilon_{n}-\varepsilon_{q}-\varepsilon_{r}\right)\left(\varepsilon_{m}-\varepsilon_{r}\right)}\right] \text {. }
\end{aligned}
$$


We can now extend the sums over $m$ and $n$, as well as $q$ and $r$ to all values in Eq. (S30) and combine terms in successive lines to obtain:

$$
\begin{aligned}
E_{D 1}^{(3,1)} & =\frac{1}{2} \sum_{m, n}^{o c c} \sum_{p, q, r}^{v i r t} R\left[\{(p q \mid n r)-(p r \mid n q)\} \frac{\left\langle m\left|\hat{h}_{S O}\right| p\right\rangle\left\langle r\left|\hat{h}_{S O}\right| n\right\rangle\left\langle q\left|\hat{h}_{S O}\right| m\right\rangle}{\left(\varepsilon_{m}+\varepsilon_{n}-\varepsilon_{q}-\varepsilon_{r}\right)}\right]\left[\frac{1}{\left(\varepsilon_{m}-\varepsilon_{p}\right)\left(\varepsilon_{m}-\varepsilon_{q}\right)}+\frac{1}{\left(\varepsilon_{m}-\varepsilon_{p}\right)\left(\varepsilon_{n}-\varepsilon_{r}\right)}\right] \\
& -\frac{1}{2} \sum_{m, n}^{o c c} \sum_{p, q, r}^{v i r t} R\left[\{(p q \mid n r)-(p r \mid n q)\} \frac{\left\langle m\left|\hat{h}_{S O}\right| p\right\rangle\left\langle r\left|\hat{h}_{S O}\right| m\right\rangle\left\langle q\left|\hat{h}_{S O}\right| n\right\rangle}{\left(\varepsilon_{m}+\varepsilon_{n}-\varepsilon_{q}-\varepsilon_{r}\right)}\right]\left[\frac{1}{\left(\varepsilon_{m}-\varepsilon_{p}\right)\left(\varepsilon_{n}-\varepsilon_{q}\right)}+\frac{1}{\left(\varepsilon_{m}-\varepsilon_{p}\right)\left(\varepsilon_{m}-\varepsilon_{r}\right)}\right] \\
& +\frac{1}{2} \sum_{m, n}^{o c c} \sum_{p, q, r}^{v i r t} R\left[\{(m q \mid p r)-(m r \mid p q)\} \frac{\left\langle n\left|\hat{h}_{S O}\right| p\right\rangle\left\langle r\left|\hat{h}_{S O}\right| n\right\rangle\left\langle q\left|\hat{h}_{S O}\right| m\right\rangle}{\left(\varepsilon_{m}+\varepsilon_{n}-\varepsilon_{q}-\varepsilon_{r}\right)}\right]\left[\frac{1}{\left(\varepsilon_{n}-\varepsilon_{p}\right)\left(\varepsilon_{m}-\varepsilon_{q}\right)}+\frac{1}{\left(\varepsilon_{n}-\varepsilon_{p}\right)\left(\varepsilon_{n}-\varepsilon_{r}\right)}\right] \\
& -\frac{1}{2} \sum_{m, n}^{o c c} \sum_{p, q, r}^{v i r t} R\left[\{(m q \mid p r)-(m r \mid p q)\} \frac{\left\langle n\left|\hat{h}_{S O}\right| p\right\rangle\left\langle r\left|\hat{h}_{S O}\right| m\right\rangle\left\langle q\left|\hat{h}_{S O}\right| n\right\rangle}{\left(\varepsilon_{m}+\varepsilon_{n}-\varepsilon_{q}-\varepsilon_{r}\right)}\right]\left[\frac{1}{\left(\varepsilon_{n}-\varepsilon_{p}\right)\left(\varepsilon_{n}-\varepsilon_{q}\right)}+\frac{1}{\left(\varepsilon_{n}-\varepsilon_{p}\right)\left(\varepsilon_{m}-\varepsilon_{r}\right)}\right] \\
& +\frac{1}{2} \sum_{l, m, n}^{o c c} \sum_{q, r}^{v i r t} R\left[\{(m r \mid n l)-(m l \mid n r)\} \frac{\left\langle l\left|\hat{h}_{S O}\right| q\right\rangle\left\langle r\left|\hat{h}_{S O}\right| n\right\rangle\left\langle q\left|\hat{h}_{S O}\right| m\right\rangle}{\left(\varepsilon_{m}+\varepsilon_{n}-\varepsilon_{q}-\varepsilon_{r}\right)}\right]\left[\frac{1}{\left(\varepsilon_{l}-\varepsilon_{q}\right)\left(\varepsilon_{m}-\varepsilon_{q}\right)}+\frac{1}{\left(\varepsilon_{l}-\varepsilon_{q}\right)\left(\varepsilon_{n}-\varepsilon_{r}\right)}\right] \\
& -\frac{1}{2} \sum_{l, m, n}^{o c c} \sum_{q, r}^{v i r t} R\left[\{(m r \mid n l)-(m l \mid n r)\} \frac{\left\langle l\left|\hat{h}_{S O}\right| q\right\rangle\left\langle r\left|\hat{h}_{S O}\right| m\right\rangle\left\langle q\left|\hat{h}_{S O}\right| n\right\rangle}{\left(\varepsilon_{m}+\varepsilon_{n}-\varepsilon_{q}-\varepsilon_{r}\right)}\right]\left[\frac{1}{\left(\varepsilon_{l}-\varepsilon_{q}\right)\left(\varepsilon_{n}-\varepsilon_{q}\right)}+\frac{1}{\left(\varepsilon_{l}-\varepsilon_{q}\right)\left(\varepsilon_{m}-\varepsilon_{r}\right)}\right] \\
& +\frac{1}{2} \sum_{l, m, n}^{o c c} \sum_{q, r}^{v i r t} R\left[\{(m l \mid n q)-(m q \mid n l)\} \frac{\left\langle l\left|\hat{h}_{S O}\right| r\right\rangle\left\langle r\left|\hat{h}_{S O}\right| n\right\rangle\left\langle q\left|\hat{h}_{S O}\right| m\right\rangle}{\left(\varepsilon_{m}+\varepsilon_{n}-\varepsilon_{q}-\varepsilon_{r}\right)}\right]\left[\frac{1}{\left(\varepsilon_{l}-\varepsilon_{r}\right)\left(\varepsilon_{m}-\varepsilon_{q}\right)}+\frac{1}{\left(\varepsilon_{l}-\varepsilon_{r}\right)\left(\varepsilon_{n}-\varepsilon_{r}\right)}\right] \\
& -\frac{1}{2} \sum_{l, m, n}^{o c c} \sum_{q, r}^{v i r t} R\left[\{(m l \mid n q)-(m q \mid n l)\} \frac{\left\langle l\left|\hat{h}_{S O}\right| r\right\rangle\left\langle r\left|\hat{h}_{S O}\right| m\right\rangle\left\langle q\left|\hat{h}_{S O}\right| n\right\rangle}{\left(\varepsilon_{m}+\varepsilon_{n}-\varepsilon_{q}-\varepsilon_{r}\right)}\right]\left[\frac{1}{\left(\varepsilon_{l}-\varepsilon_{r}\right)\left(\varepsilon_{n}-\varepsilon_{q}\right)}+\frac{1}{\left(\varepsilon_{l}-\varepsilon_{r}\right)\left(\varepsilon_{m}-\varepsilon_{r}\right)}\right] .(S 31)
\end{aligned}
$$

Then, expressing the two fractions in each line of Eq. (S31) with a common denominator yields:

$$
\begin{aligned}
E_{D 1}^{(3,1)} & =\frac{1}{2} \sum_{m, n}^{o c c} \sum_{p, q, r}^{v i r t} R\left[\{(p q \mid n r)-(p r \mid n q)\} \frac{\left\langle m\left|\hat{h}_{S O}\right| p\right\rangle\left\langle r\left|\hat{h}_{S O}\right| n\right\rangle\left\langle q\left|\hat{h}_{S O}\right| m\right\rangle}{\left(\varepsilon_{m}-\varepsilon_{p}\right)\left(\varepsilon_{m}-\varepsilon_{q}\right)\left(\varepsilon_{n}-\varepsilon_{r}\right)}\right] \\
& -\frac{1}{2} \sum_{m, n}^{o c c} \sum_{p, q, r}^{v i r t} R\left[\{(p q \mid n r)-(p r \mid n q)\} \frac{\left\langle m\left|\hat{h}_{S O}\right| p\right\rangle\left\langle r\left|\hat{h}_{S O}\right| m\right\rangle\left\langle q\left|\hat{h}_{S O}\right| n\right\rangle}{\left(\varepsilon_{m}-\varepsilon_{p}\right)\left(\varepsilon_{n}-\varepsilon_{q}\right)\left(\varepsilon_{m}-\varepsilon_{r}\right)}\right] \\
& +\frac{1}{2} \sum_{m, n}^{o c c} \sum_{p, q, r}^{v i r t} R\left[\{(m q \mid p r)-(m r \mid p q)\} \frac{\left\langle n\left|\hat{h}_{S O}\right| p\right\rangle\left\langle r\left|\hat{h}_{S O}\right| n\right\rangle\left\langle q\left|\hat{h}_{S O}\right| m\right\rangle}{\left(\varepsilon_{n}-\varepsilon_{p}\right)\left(\varepsilon_{m}-\varepsilon_{q}\right)\left(\varepsilon_{n}-\varepsilon_{r}\right)}\right] \\
& -\frac{1}{2} \sum_{m, n}^{o c c} \sum_{p, q, r}^{v i r t} R\left[\{(m q \mid p r)-(m r \mid p q)\} \frac{\left\langle n\left|\hat{h}_{S O}\right| p\right\rangle\left\langle r\left|\hat{h}_{S O}\right| m\right\rangle\left\langle q\left|\hat{h}_{S O}\right| n\right\rangle}{\left(\varepsilon_{n}-\varepsilon_{p}\right)\left(\varepsilon_{n}-\varepsilon_{q}\right)\left(\varepsilon_{m}-\varepsilon_{r}\right)}\right] \\
& +\frac{1}{2} \sum_{l, m, n}^{o c c} \sum_{q, r}^{v i r t} R\left[\{(m r \mid n l)-(m l \mid n r)\} \frac{\left\langle l\left|\hat{h}_{S O}\right| q\right\rangle\left\langle r\left|\hat{h}_{S O}\right| n\right\rangle\left\langle q\left|\hat{h}_{S O}\right| m\right\rangle}{\left(\varepsilon_{l}-\varepsilon_{q}\right)\left(\varepsilon_{m}-\varepsilon_{q}\right)\left(\varepsilon_{n}-\varepsilon_{r}\right)}\right] \\
& -\frac{1}{2} \sum_{l, m, n}^{o c c} \sum_{q, r}^{\text {virt }} R\left[\{(m r \mid n l)-(m l \mid n r)\} \frac{\left\langle l\left|\hat{h}_{S O}\right| q\right\rangle\left\langle r\left|\hat{h}_{S O}\right| m\right\rangle\left\langle q\left|\hat{h}_{S O}\right| n\right\rangle}{\left(\varepsilon_{l}-\varepsilon_{q}\right)\left(\varepsilon_{n}-\varepsilon_{q}\right)\left(\varepsilon_{m}-\varepsilon_{r}\right)}\right] \\
& +\frac{1}{2} \sum_{l, m, n}^{o c c} \sum_{q, r}^{v i r t} R\left[\{(m l \mid n q)-(m q \mid n l)\} \frac{\left\langle l\left|\hat{h}_{S O}\right| r\right\rangle\left\langle r\left|\hat{h}_{S O}\right| n\right\rangle\left\langle q\left|\hat{h}_{S O}\right| m\right\rangle}{\left(\varepsilon_{l}-\varepsilon_{r}\right)\left(\varepsilon_{m}-\varepsilon_{q}\right)\left(\varepsilon_{n}-\varepsilon_{r}\right)}\right] \\
& -\frac{1}{2} \sum_{l, m, n} \sum_{q, r}^{o c c} R\left[\{(m l \mid n q)-(m q \mid n l)\} \frac{\left\langle l\left|\hat{h}_{S O}\right| r\right\rangle\left\langle r\left|\hat{h}_{S O}\right| m\right\rangle\left\langle q\left|\hat{h}_{S O}\right| n\right\rangle}{\left(\varepsilon_{l}-\varepsilon_{r}\right)\left(\varepsilon_{n}-\varepsilon_{q}\right)\left(\varepsilon_{m}-\varepsilon_{r}\right)}\right] .
\end{aligned}
$$


Switching the indices $r \leftrightarrow q$ in lines 2, as well as 4 and $m \leftrightarrow n$ in lines 6 and 8 of Eq. (S32) we obtain:

$$
\begin{aligned}
E_{D 1}^{(3,1)} & =\sum_{m, n}^{o c c} \sum_{p, q, r}^{\text {virt }} R\left[\{(p q \mid n r)-(p r \mid n q)\} \frac{\left\langle m\left|\hat{h}_{S O}\right| p\right\rangle\left\langle r\left|\hat{h}_{S O}\right| n\right\rangle\left\langle q\left|\hat{h}_{S O}\right| m\right\rangle}{\left(\varepsilon_{m}-\varepsilon_{p}\right)\left(\varepsilon_{m}-\varepsilon_{q}\right)\left(\varepsilon_{n}-\varepsilon_{r}\right)}\right] \\
& +\sum_{m, n}^{o c c} \sum_{p, q, r}^{v i r t} R\left[\{(m q \mid p r)-(m r \mid p q)\} \frac{\left\langle n\left|\hat{h}_{S O}\right| p\right\rangle\left\langle r\left|\hat{h}_{S O}\right| n\right\rangle\left\langle q\left|\hat{h}_{S O}\right| m\right\rangle}{\left(\varepsilon_{n}-\varepsilon_{p}\right)\left(\varepsilon_{m}-\varepsilon_{q}\right)\left(\varepsilon_{n}-\varepsilon_{r}\right)}\right] \\
& +\sum_{l, m, n} \sum_{q, r}^{v i r t} R\left[\{(m r \mid n l)-(m l \mid n r)\} \frac{\left\langle l\left|\hat{h}_{S O}\right| q\right\rangle\left\langle r\left|\hat{h}_{S O}\right| n\right\rangle\left\langle q\left|\hat{h}_{S O}\right| m\right\rangle}{\left(\varepsilon_{l}-\varepsilon_{q}\right)\left(\varepsilon_{m}-\varepsilon_{q}\right)\left(\varepsilon_{n}-\varepsilon_{r}\right)}\right] \\
& +\sum_{l, m, n}^{\text {occ }} \sum_{q, r}^{\text {virt }} R\left[\{(m l \mid n q)-(m q \mid n l)\} \frac{\left\langle l\left|\hat{h}_{S O}\right| r\right\rangle\left\langle r\left|\hat{h}_{S O}\right| n\right\rangle\left\langle q\left|\hat{h}_{S O}\right| m\right\rangle}{\left(\varepsilon_{l}-\varepsilon_{r}\right)\left(\varepsilon_{m}-\varepsilon_{q}\right)\left(\varepsilon_{n}-\varepsilon_{r}\right)}\right] .
\end{aligned}
$$

A switch of the indices $r \leftrightarrow q$ and $m \leftrightarrow n$ in lines 2 and 4 of Eq. (S33) gives:

$$
\begin{aligned}
E_{D 1}^{(3,1)} & =2 \sum_{m, n}^{o c c} \sum_{p, q, r}^{\text {virt }} R\left[\{(p q \mid n r)-(p r \mid n q)\} \frac{\left\langle m\left|\hat{h}_{S O}\right| p\right\rangle\left\langle r\left|\hat{h}_{S O}\right| n\right\rangle\left\langle q\left|\hat{h}_{S O}\right| m\right\rangle}{\left(\varepsilon_{m}-\varepsilon_{p}\right)\left(\varepsilon_{m}-\varepsilon_{q}\right)\left(\varepsilon_{n}-\varepsilon_{r}\right)}\right] \\
& +2 \sum_{l, m, n}^{\text {occ }} \sum_{q, r}^{\text {virt }} R\left[\{(m r \mid n l)-(m l \mid n r)\} \frac{\left\langle l\left|\hat{h}_{S O}\right| q\right\rangle\left\langle r\left|\hat{h}_{S O}\right| n\right\rangle\left\langle q\left|\hat{h}_{S O}\right| m\right\rangle}{\left(\varepsilon_{l}-\varepsilon_{q}\right)\left(\varepsilon_{m}-\varepsilon_{q}\right)\left(\varepsilon_{n}-\varepsilon_{r}\right)}\right] .
\end{aligned}
$$

Finally, in the first line of Eq. (S34) we switch the indices $p \rightarrow r, q \rightarrow p, r \rightarrow q$ and in the second lines $r \rightarrow q, q \rightarrow p, l \rightarrow o$, such that we obtain:

$$
\begin{aligned}
E_{D 1}^{(3,1)} & =2 \sum_{m, n}^{o c c} \sum_{p, q, r}^{\text {virt }} R\left[\{(r p \mid n q)-(r q \mid n p)\} \frac{\left\langle m\left|\hat{h}_{S O}\right| r\right\rangle\left\langle q\left|\hat{h}_{S O}\right| n\right\rangle\left\langle p\left|\hat{h}_{S O}\right| m\right\rangle}{\left(\varepsilon_{m}-\varepsilon_{r}\right)\left(\varepsilon_{n}-\varepsilon_{q}\right)\left(\varepsilon_{m}-\varepsilon_{p}\right)}\right] \\
& +2 \sum_{m, n, o}^{o c c} \sum_{p, q}^{v i r t} R\left[\{(m q \mid n o)-(m o \mid n q)\} \frac{\left\langle o\left|\hat{h}_{S O}\right| p\right\rangle\left\langle q \mid \hat{h}_{S O} n\right\rangle\left\langle p\left|\hat{h}_{S O}\right| m\right\rangle}{\left(\varepsilon_{o}-\varepsilon_{p}\right)\left(\varepsilon_{n}-\varepsilon_{q}\right)\left(\varepsilon_{m}-\varepsilon_{p}\right)}\right]
\end{aligned}
$$

\section{The Contribution $E_{D 2}^{(3,1)}$}

Returning to Eq. (S27), we require integrals involving pairs of doubly-excited determinants and the SOC operator. We evaluate them from the Slater-Condon rules to obtain:

$$
\left\langle\psi_{l m}^{q r}\left|\hat{h}_{S O}\right| \psi_{n o}^{s t}\right\rangle=\delta_{n, l} \delta_{s, q} \delta_{o, m}\left\langle r\left|\hat{h}_{S O}\right| t\right\rangle-\delta_{n, l} \delta_{s, q} \delta_{t, r}\left\langle o\left|\hat{h}_{S O}\right| m\right\rangle+\delta_{n, l} \delta_{t, r} \delta_{o, m}\left\langle q\left|\hat{h}_{S O}\right| s\right\rangle-\delta_{s, q} \delta_{t, r} \delta_{o, m}\left\langle n\left|\hat{h}_{S O}\right| l\right\rangle
$$

We also require integrals involving a doubly-excited determinant and the electron fluctuation potential, which is expressed as follows:

$$
\left\langle\psi^{(0,0)}\left|\hat{H}^{(0,1)}\right| \psi_{m n}^{q r}\right\rangle=(m q \mid n r)-(m r \mid n q) .
$$

Substituting Eqs. (S3b), (S9a), (S9b), (S36) and (S37) in Eq. (S27) gives:

$$
\begin{aligned}
E_{D 2}^{(3,1)} & =\sum_{l, o}^{o c c} \sum_{m>n}^{o c c} \sum_{p, s}^{\text {virt }} \sum_{q>r}^{\text {virt }} 2 R\left[\{ ( m q | n r ) - ( m r | n q ) \} \left\{\delta_{p, q} \delta_{l, m}\left\langle r\left|\hat{h}_{S O}\right| n\right\rangle\right.\right. \\
& \left.+\delta_{p, r} \delta_{l, n}\left\langle q\left|\hat{h}_{S O}\right| m\right\rangle-\delta_{p, q} \delta_{l, n}\left\langle r\left|\hat{h}_{S O}\right| m\right\rangle-\delta_{p, r} \delta_{l, m}\left\langle q\left|\hat{h}_{S O}\right| n\right\rangle\right\} \\
& \left.\times \frac{\left\{\delta_{o, l}\left\langle p\left|\hat{h}_{S O}\right| s\right\rangle-\delta_{p, s}\left\langle o\left|\hat{h}_{S O}\right| l\right\rangle\right\}\left\langle s\left|\hat{h}_{S O}\right| o\right\rangle}{\left(\varepsilon_{m}+\varepsilon_{n}-\varepsilon_{q}-\varepsilon_{r}\right)\left(\varepsilon_{l}-\varepsilon_{p}\right)\left(\varepsilon_{o}-\varepsilon_{s}\right)}\right] \\
& +\sum_{k} \sum_{l>m} \sum_{n, o} \sum_{p} \sum_{q>r}^{\text {occ }} \sum_{S, t}^{\text {virt }} 2 R\left[\{ ( l q | m r ) - ( l r | m q ) \} \left\{\delta_{n, l} \delta_{s, q} \delta_{o, m}\left\langle r\left|\hat{h}_{S O}\right| t\right\rangle\right.\right. \\
& \left.-\delta_{n, l} \delta_{s, q} \delta_{t, r}\left\langle o\left|\hat{h}_{S O}\right| m\right\rangle+\delta_{n, l} \delta_{t, r} \delta_{o, m}\left\langle q\left|\hat{h}_{S O}\right| s\right\rangle-\delta_{s, q} \delta_{t, r} \delta_{o, m}\left\langle n\left|\hat{h}_{S O}\right| l\right\rangle\right\} \\
& \left.\times \frac{\left\{\delta_{p, s} \delta_{k, n}\left\langle t\left|\hat{h}_{S O}\right| o\right\rangle+\delta_{p, t} \delta_{k, o}\left\langle s\left|\hat{h}_{S O}\right| n\right\rangle-\delta_{p, s} \delta_{k, o}\left\langle t\left|\hat{h}_{S O}\right| n\right\rangle-\delta_{p, t} \delta_{k, n}\left\langle s\left|\hat{h}_{S O}\right| o\right\rangle\right\}\left\langle p\left|\hat{h}_{S O}\right| k\right\rangle}{\left(\varepsilon_{l}+\varepsilon_{m}-\varepsilon_{q}-\varepsilon_{r}\right)\left(\varepsilon_{n}+\varepsilon_{o}-\varepsilon_{s}-\varepsilon_{t}\right)\left(\varepsilon_{k}-\varepsilon_{p}\right)}\right]
\end{aligned}
$$


Distributing the Kronecker deltas in the first, second, third and last lines Eq. (S38), we obtain:

$$
\begin{aligned}
& E_{D 2}^{(3,1)}=\sum_{m>n}^{o c c} \sum_{\substack{q>r \\
s>r}}^{v i r t} 2 R\left[\{(m q \mid n r)-(m r \mid n q)\} \frac{\left\langle r\left|\hat{h}_{S O}\right| n\right\rangle\left\langle q\left|\hat{h}_{S O}\right| s\right\rangle\left\langle s\left|\hat{h}_{S O}\right| m\right\rangle}{\left(\varepsilon_{m}+\varepsilon_{n}-\varepsilon_{q}-\varepsilon_{r}\right)\left(\varepsilon_{m}-\varepsilon_{q}\right)\left(\varepsilon_{m}-\varepsilon_{s}\right)}\right] \\
& -\sum_{o}^{o c c} \sum_{m>n}^{o c c} \sum_{q>r}^{v i r t} 2 R\left[\{(m q \mid n r)-(m r \mid n q)\} \frac{\left\langle r\left|\hat{h}_{S O}\right| n\right\rangle\left\langle o\left|\hat{h}_{S O}\right| m\right\rangle\left\langle q\left|\hat{h}_{S O}\right| o\right\rangle}{\left(\varepsilon_{m}+\varepsilon_{n}-\varepsilon_{q}-\varepsilon_{r}\right)\left(\varepsilon_{m}-\varepsilon_{q}\right)\left(\varepsilon_{o}-\varepsilon_{q}\right)}\right] \\
& +\sum_{m>n}^{o c c} \sum_{s}^{\text {virt }} \sum_{q>r}^{v i r t} 2 R\left[\{(m q \mid n r)-(m r \mid n q)\} \frac{\left\langle q\left|\hat{h}_{S O}\right| m\right\rangle\left\langle r\left|\hat{h}_{S O}\right| s\right\rangle\left\langle s\left|\hat{h}_{S O}\right| n\right\rangle}{\left(\varepsilon_{m}+\varepsilon_{n}-\varepsilon_{q}-\varepsilon_{r}\right)\left(\varepsilon_{n}-\varepsilon_{r}\right)\left(\varepsilon_{n}-\varepsilon_{s}\right)}\right] \\
& -\sum_{o}^{o c c} \sum_{m>n}^{o c c} \sum_{q>r}^{v i r t} 2 R\left[\{(m q \mid n r)-(m r \mid n q)\} \frac{\left\langle q\left|\hat{h}_{S O}\right| m\right\rangle\left\langle o\left|\hat{h}_{S O}\right| n\right\rangle\left\langle r\left|\hat{h}_{S O}\right| o\right\rangle}{\left(\varepsilon_{m}+\varepsilon_{n}-\varepsilon_{q}-\varepsilon_{r}\right)\left(\varepsilon_{n}-\varepsilon_{r}\right)\left(\varepsilon_{o}-\varepsilon_{r}\right)}\right] \\
& -\sum_{m>n}^{o c c} \sum_{S}^{\text {virt }} \sum_{q>r}^{v i r t} 2 R\left[\{(m q \mid n r)-(m r \mid n q)\} \frac{\left\langle r\left|\hat{h}_{S O}\right| m\right\rangle\left\langle q\left|\hat{h}_{S O}\right| s\right\rangle\left\langle s\left|\hat{h}_{S O}\right| n\right\rangle}{\left(\varepsilon_{m}+\varepsilon_{n}-\varepsilon_{q}-\varepsilon_{r}\right)\left(\varepsilon_{n}-\varepsilon_{q}\right)\left(\varepsilon_{n}-\varepsilon_{S}\right)}\right] \\
& +\sum_{o}^{o c c} \sum_{m>n}^{o c c} \sum_{q>r}^{v i r t} 2 R\left[\{(m q \mid n r)-(m r \mid n q)\} \frac{\left\langle r\left|\hat{h}_{S O}\right| m\right\rangle\left\langle o\left|\hat{h}_{S O}\right| n\right\rangle\left\langle q\left|\hat{h}_{S O}\right| o\right\rangle}{\left(\varepsilon_{m}+\varepsilon_{n}-\varepsilon_{q}-\varepsilon_{r}\right)\left(\varepsilon_{n}-\varepsilon_{q}\right)\left(\varepsilon_{o}-\varepsilon_{q}\right)}\right] \\
& -\sum_{m>n}^{o c c} \sum_{s}^{\text {virt }} \sum_{q>r}^{\text {virt }} 2 R\left[\{(m q \mid n r)-(m r \mid n q)\} \frac{\left\langle q\left|\hat{h}_{S O}\right| n\right\rangle\left\langle r\left|\hat{h}_{S O}\right| s\right\rangle\left\langle s\left|\hat{h}_{S O}\right| m\right\rangle}{\left(\varepsilon_{m}+\varepsilon_{n}-\varepsilon_{q}-\varepsilon_{r}\right)\left(\varepsilon_{m}-\varepsilon_{r}\right)\left(\varepsilon_{m}-\varepsilon_{s}\right)}\right] \\
& +\sum_{o}^{o c c} \sum_{m>n}^{o c c} \sum_{q>r}^{v i r t} 2 R\left[\{(m q \mid n r)-(m r \mid n q)\} \frac{\left\langle q\left|\hat{h}_{S O}\right| n\right\rangle\left\langle o\left|\hat{h}_{S O}\right| m\right\rangle\left\langle r\left|\hat{h}_{S O}\right| o\right\rangle}{\left(\varepsilon_{m}+\varepsilon_{n}-\varepsilon_{q}-\varepsilon_{r}\right)\left(\varepsilon_{m}-\varepsilon_{r}\right)\left(\varepsilon_{o}-\varepsilon_{r}\right)}\right] \\
& +\sum_{l>m}^{o c c} \sum_{n, o}^{o c c} \sum_{q>r}^{\text {virt }} \sum_{s, t}^{\text {virt }} 2 R\left[\{ ( l q | m r ) - ( l r | m q ) \} \left\{\boldsymbol{\delta}_{n, l} \boldsymbol{\delta}_{s, q} \boldsymbol{\delta}_{o, m}\left\langle r\left|\hat{h}_{S O}\right| t\right\rangle\right.\right. \\
& \left.-\delta_{n, l} \delta_{s, q} \delta_{t, r}\left\langle o\left|\hat{h}_{S O}\right| m\right\rangle+\delta_{n, l} \delta_{t, r} \delta_{o, m}\left\langle q\left|\hat{h}_{S O}\right| s\right\rangle-\delta_{s, q} \delta_{t, r} \delta_{o, m}\left\langle n\left|\hat{h}_{S O}\right| l\right\rangle\right\} \frac{1}{\left(\varepsilon_{l}+\varepsilon_{m}-\varepsilon_{q}-\varepsilon_{r}\right)\left(\varepsilon_{n}+\varepsilon_{o}-\varepsilon_{s}-\varepsilon_{t}\right)} \\
& \left.\times\left\{\frac{\left\langle t\left|\hat{h}_{S O}\right| o\right\rangle\left\langle s\left|\hat{h}_{S O}\right| n\right\rangle}{\varepsilon_{n}-\varepsilon_{S}}+\frac{\left\langle s\left|\hat{h}_{S O}\right| n\right\rangle\left\langle t\left|\hat{h}_{S O}\right| o\right\rangle}{\varepsilon_{o}-\varepsilon_{t}}-\frac{\left\langle s\left|\hat{h}_{S O}\right| o\right\rangle\left\langle t\left|\hat{h}_{S O}\right| n\right\rangle}{\varepsilon_{o}-\varepsilon_{S}}-\frac{\left\langle t\left|\hat{h}_{S O}\right| n\right\rangle\left\langle s\left|\hat{h}_{S O}\right| o\right\rangle}{\varepsilon_{n}-\varepsilon_{t}}\right\}\right] .
\end{aligned}
$$


Expressing the first and second terms as well as the third and fourth terms in the last line of Eq. (S39) with a common denominator gives:

$$
\begin{aligned}
& E_{D 2}^{(3,1)}=\sum_{m>n}^{o c c} \sum_{S}^{\text {virt }} \sum_{q>r}^{v i r t} 2 R\left[\{(m q \mid n r)-(m r \mid n q)\} \frac{\left\langle r\left|\hat{h}_{S O}\right| n\right\rangle\left\langle q\left|\hat{h}_{S O}\right| s\right\rangle\left\langle s\left|\hat{h}_{S O}\right| m\right\rangle}{\left(\varepsilon_{m}+\varepsilon_{n}-\varepsilon_{q}-\varepsilon_{r}\right)\left(\varepsilon_{m}-\varepsilon_{q}\right)\left(\varepsilon_{m}-\varepsilon_{s}\right)}\right] \\
& -\sum_{o}^{o c c} \sum_{m>n}^{o c c} \sum_{q>r}^{v i r t} 2 R\left[\{(m q \mid n r)-(m r \mid n q)\} \frac{\left\langle r\left|\hat{h}_{S O}\right| n\right\rangle\left\langle o\left|\hat{h}_{S O}\right| m\right\rangle\left\langle q\left|\hat{h}_{S O}\right| o\right\rangle}{\left(\varepsilon_{m}+\varepsilon_{n}-\varepsilon_{q}-\varepsilon_{r}\right)\left(\varepsilon_{m}-\varepsilon_{q}\right)\left(\varepsilon_{o}-\varepsilon_{q}\right)}\right] \\
& +\sum_{m>n}^{o c c} \sum_{s}^{\text {virt }} \sum_{q>r}^{\text {virt }} 2 R\left[\{(m q \mid n r)-(m r \mid n q)\} \frac{\left\langle q\left|\hat{h}_{S O}\right| m\right\rangle\left\langle r\left|\hat{h}_{S O}\right| s\right\rangle\left\langle s\left|\hat{h}_{S O}\right| n\right\rangle}{\left(\varepsilon_{m}+\varepsilon_{n}-\varepsilon_{q}-\varepsilon_{r}\right)\left(\varepsilon_{n}-\varepsilon_{r}\right)\left(\varepsilon_{n}-\varepsilon_{s}\right)}\right] \\
& -\sum_{o}^{o c c} \sum_{m>n}^{o c c} \sum_{q>r}^{v i r t} 2 R\left[\{(m q \mid n r)-(m r \mid n q)\} \frac{\left\langle q\left|\hat{h}_{S O}\right| m\right\rangle\left\langle o\left|\hat{h}_{S O}\right| n\right\rangle\left\langle r\left|\hat{h}_{S O}\right| o\right\rangle}{\left(\varepsilon_{m}+\varepsilon_{n}-\varepsilon_{q}-\varepsilon_{r}\right)\left(\varepsilon_{n}-\varepsilon_{r}\right)\left(\varepsilon_{o}-\varepsilon_{r}\right)}\right] \\
& -\sum_{m>n}^{o c c} \sum_{s}^{\text {virt }} \sum_{q>r}^{\text {virt }} 2 R\left[\{(m q \mid n r)-(m r \mid n q)\} \frac{\left\langle r\left|\hat{h}_{S O}\right| m\right\rangle\left\langle q\left|\hat{h}_{S O}\right| s\right\rangle\left\langle s\left|\hat{h}_{S O}\right| n\right\rangle}{\left(\varepsilon_{m}+\varepsilon_{n}-\varepsilon_{q}-\varepsilon_{r}\right)\left(\varepsilon_{n}-\varepsilon_{q}\right)\left(\varepsilon_{n}-\varepsilon_{s}\right)}\right] \\
& +\sum_{o}^{o c c} \sum_{m>n}^{o c c} \sum_{q>r}^{v i r t} 2 R\left[\{(m q \mid n r)-(m r \mid n q)\} \frac{\left\langle r\left|\hat{h}_{S O}\right| m\right\rangle\left\langle o\left|\hat{h}_{S O}\right| n\right\rangle\left\langle q\left|\hat{h}_{S O}\right| o\right\rangle}{\left(\varepsilon_{m}+\varepsilon_{n}-\varepsilon_{q}-\varepsilon_{r}\right)\left(\varepsilon_{n}-\varepsilon_{q}\right)\left(\varepsilon_{o}-\varepsilon_{q}\right)}\right] \\
& -\sum_{m>n}^{o c c} \sum_{S}^{\text {virt }} \sum_{q>r}^{\text {virt }} 2 R\left[\{(m q \mid n r)-(m r \mid n q)\} \frac{\left\langle q\left|\hat{h}_{S O}\right| n\right\rangle\left\langle r\left|\hat{h}_{S O}\right| s\right\rangle\left\langle s\left|\hat{h}_{S O}\right| m\right\rangle}{\left(\varepsilon_{m}+\varepsilon_{n}-\varepsilon_{q}-\varepsilon_{r}\right)\left(\varepsilon_{m}-\varepsilon_{r}\right)\left(\varepsilon_{m}-\varepsilon_{s}\right)}\right] \\
& +\sum_{o}^{o c c} \sum_{m>n}^{o c c} \sum_{q>r}^{v i r t} 2 R\left[\{(m q \mid n r)-(m r \mid n q)\} \frac{\left\langle q\left|\hat{h}_{S O}\right| n\right\rangle\left\langle o\left|\hat{h}_{S O}\right| m\right\rangle\left\langle r\left|\hat{h}_{S O}\right| o\right\rangle}{\left(\varepsilon_{m}+\varepsilon_{n}-\varepsilon_{q}-\varepsilon_{r}\right)\left(\varepsilon_{m}-\varepsilon_{r}\right)\left(\varepsilon_{o}-\varepsilon_{r}\right)}\right] \\
& +\sum_{l>m}^{o c c} \sum_{n, o}^{o c c} \sum_{q>r}^{\text {virt }} \sum_{s, t}^{\text {vit }} 2 R\left[\{ ( l q | m r ) - ( l r | m q ) \} \left\{\delta_{n, l} \delta_{s, q} \delta_{o, m}\left\langle r\left|\hat{h}_{S O}\right| t\right\rangle\right.\right. \\
& \left.-\delta_{n, l} \delta_{s, q} \delta_{t, r}\left\langle o\left|\hat{h}_{S O}\right| m\right\rangle+\delta_{n, l} \delta_{t, r} \delta_{o, m}\left\langle q\left|\hat{h}_{S O}\right| s\right\rangle-\delta_{s, q} \delta_{t, r} \delta_{o, m}\left\langle n\left|\hat{h}_{S O}\right| l\right\rangle\right\} \frac{1}{\left(\varepsilon_{l}+\varepsilon_{m}-\varepsilon_{q}-\varepsilon_{r}\right)} \\
& \left.\times\left\{\frac{\left\langle t\left|\hat{h}_{S O}\right| o\right\rangle\left\langle s\left|\hat{h}_{S O}\right| n\right\rangle}{\left(\varepsilon_{n}-\varepsilon_{s}\right)\left(\varepsilon_{o}-\varepsilon_{t}\right)}-\frac{\left\langle s\left|\hat{h}_{S O}\right| o\right\rangle\left\langle t\left|\hat{h}_{S O}\right| n\right\rangle}{\left(\varepsilon_{o}-\varepsilon_{S}\right)\left(\varepsilon_{n}-\varepsilon_{t}\right)}\right\}\right] .
\end{aligned}
$$


Now evaluating the Kronecker deltas in the eighth and ninth lines of Eq. (S40), we obtain:

$$
\begin{aligned}
& E_{D 2}^{(3,1)}=\sum_{m>n}^{o c c} \sum_{s}^{\text {virt }} \sum_{q>r}^{v i r t} 2 R\left[\{(m q \mid n r)-(m r \mid n q)\} \frac{\left\langle r\left|\hat{h}_{S O}\right| n\right\rangle\left\langle q\left|\hat{h}_{S O}\right| s\right\rangle\left\langle s\left|\hat{h}_{S O}\right| m\right\rangle}{\left(\varepsilon_{m}+\varepsilon_{n}-\varepsilon_{q}-\varepsilon_{r}\right)\left(\varepsilon_{m}-\varepsilon_{q}\right)\left(\varepsilon_{m}-\varepsilon_{s}\right)}\right] \\
& -\sum_{o}^{o c c} \sum_{m>n}^{o c c} \sum_{q>r}^{v i r t} 2 R\left[\{(m q \mid n r)-(m r \mid n q)\} \frac{\left\langle r\left|\hat{h}_{S O}\right| n\right\rangle\left\langle o\left|\hat{h}_{S O}\right| m\right\rangle\left\langle q\left|\hat{h}_{S O}\right| o\right\rangle}{\left(\varepsilon_{m}+\varepsilon_{n}-\varepsilon_{q}-\varepsilon_{r}\right)\left(\varepsilon_{m}-\varepsilon_{q}\right)\left(\varepsilon_{o}-\varepsilon_{q}\right)}\right] \\
& +\sum_{m>n}^{o c c} \sum_{S}^{\text {virt }} \sum_{q>r}^{\text {virt }} 2 R\left[\{(m q \mid n r)-(m r \mid n q)\} \frac{\left\langle q\left|\hat{h}_{S O}\right| m\right\rangle\left\langle r\left|\hat{h}_{S O}\right| s\right\rangle\left\langle s\left|\hat{h}_{S O}\right| n\right\rangle}{\left(\varepsilon_{m}+\varepsilon_{n}-\varepsilon_{q}-\varepsilon_{r}\right)\left(\varepsilon_{n}-\varepsilon_{r}\right)\left(\varepsilon_{n}-\varepsilon_{s}\right)}\right] \\
& -\sum_{o}^{o c c} \sum_{m>n}^{o c c} \sum_{q>r}^{v i r t} 2 R\left[\{(m q \mid n r)-(m r \mid n q)\} \frac{\left\langle q\left|\hat{h}_{S O}\right| m\right\rangle\left\langle o\left|\hat{h}_{S O}\right| n\right\rangle\left\langle r\left|\hat{h}_{S O}\right| o\right\rangle}{\left(\varepsilon_{m}+\varepsilon_{n}-\varepsilon_{q}-\varepsilon_{r}\right)\left(\varepsilon_{n}-\varepsilon_{r}\right)\left(\varepsilon_{o}-\varepsilon_{r}\right)}\right] \\
& -\sum_{m>n}^{o c c} \sum_{s}^{\text {virt }} \sum_{q>r}^{\text {virt }} 2 R\left[\{(m q \mid n r)-(m r \mid n q)\} \frac{\left\langle r\left|\hat{h}_{S O}\right| m\right\rangle\left\langle q\left|\hat{h}_{S O}\right| s\right\rangle\left\langle s\left|\hat{h}_{S O}\right| n\right\rangle}{\left(\varepsilon_{m}+\varepsilon_{n}-\varepsilon_{q}-\varepsilon_{r}\right)\left(\varepsilon_{n}-\varepsilon_{q}\right)\left(\varepsilon_{n}-\varepsilon_{s}\right)}\right] \\
& +\sum_{o}^{o c c} \sum_{m>n}^{o c c} \sum_{q>r}^{v i r t} 2 R\left[\{(m q \mid n r)-(m r \mid n q)\} \frac{\left\langle r\left|\hat{h}_{S O}\right| m\right\rangle\left\langle o\left|\hat{h}_{S O}\right| n\right\rangle\left\langle q\left|\hat{h}_{S O}\right| o\right\rangle}{\left(\varepsilon_{m}+\varepsilon_{n}-\varepsilon_{q}-\varepsilon_{r}\right)\left(\varepsilon_{n}-\varepsilon_{q}\right)\left(\varepsilon_{o}-\varepsilon_{q}\right)}\right] \\
& -\sum_{m>n}^{o c c} \sum_{S}^{\text {virt }} \sum_{q>r}^{\text {virt }} 2 R\left[\{(m q \mid n r)-(m r \mid n q)\} \frac{\left\langle q\left|\hat{h}_{S O}\right| n\right\rangle\left\langle r\left|\hat{h}_{S O}\right| s\right\rangle\left\langle s\left|\hat{h}_{S O}\right| m\right\rangle}{\left(\varepsilon_{m}+\varepsilon_{n}-\varepsilon_{q}-\varepsilon_{r}\right)\left(\varepsilon_{m}-\varepsilon_{r}\right)\left(\varepsilon_{m}-\varepsilon_{s}\right)}\right] \\
& +\sum_{o}^{o c c} \sum_{m>n}^{o c c} \sum_{q>r}^{v i r t} 2 R\left[\{(m q \mid n r)-(m r \mid n q)\} \frac{\left\langle q\left|\hat{h}_{S O}\right| n\right\rangle\left\langle o\left|\hat{h}_{S O}\right| m\right\rangle\left\langle r\left|\hat{h}_{S O}\right| o\right\rangle}{\left(\varepsilon_{m}+\varepsilon_{n}-\varepsilon_{q}-\varepsilon_{r}\right)\left(\varepsilon_{m}-\varepsilon_{r}\right)\left(\varepsilon_{o}-\varepsilon_{r}\right)}\right] \\
& +\sum_{n>m}^{o c c} \sum_{q>r}^{v i r t} \sum_{t}^{v i r t} 2 R\left[\{(n q \mid m r)-(n r \mid m q)\} \frac{\left\langle r\left|\hat{h}_{S O}\right| t\right\rangle\left\langle t\left|\hat{h}_{S O}\right| m\right\rangle\left\langle q\left|\hat{h}_{S O}\right| n\right\rangle}{\left(\varepsilon_{n}+\varepsilon_{m}-\varepsilon_{q}-\varepsilon_{r}\right)\left(\varepsilon_{m}-\varepsilon_{t}\right)\left(\varepsilon_{n}-\varepsilon_{q}\right)}\right] \\
& -\sum_{n>m}^{o c c} \sum_{q>r}^{\text {virt }} \sum_{t}^{v i r t} 2 R\left[\{(n q \mid m r)-(n r \mid m q)\} \frac{\left\langle r\left|\hat{h}_{S O}\right| t\right\rangle\left\langle q\left|\hat{h}_{S O}\right| m\right\rangle\left\langle t\left|\hat{h}_{S O}\right| n\right\rangle}{\left(\varepsilon_{n}+\varepsilon_{m}-\varepsilon_{q}-\varepsilon_{r}\right)\left(\varepsilon_{m}-\varepsilon_{q}\right)\left(\varepsilon_{n}-\varepsilon_{t}\right)}\right] \\
& -\sum_{n>m}^{o c c} \sum_{o}^{o c c} \sum_{q>r}^{v i r t} 2 R\left[\{(n q \mid m r)-(n r \mid m q)\} \frac{\left\langle o\left|\hat{h}_{S O}\right| m\right\rangle\left\langle r\left|\hat{h}_{S O}\right| o\right\rangle\left\langle q\left|\hat{h}_{S O}\right| n\right\rangle}{\left(\varepsilon_{n}+\varepsilon_{m}-\varepsilon_{q}-\varepsilon_{r}\right)\left(\varepsilon_{n}-\varepsilon_{q}\right)\left(\varepsilon_{o}-\varepsilon_{r}\right)}\right] \\
& +\sum_{n>m}^{o c c} \sum_{o}^{o c c} \sum_{q>r}^{v i r t} 2 R\left[\{(n q \mid m r)-(n r \mid m q)\} \frac{\left\langle o\left|\hat{h}_{S O}\right| m\right\rangle\left\langle q\left|\hat{h}_{S O}\right| o\right\rangle\left\langle r\left|\hat{h}_{S O}\right| n\right\rangle}{\left(\varepsilon_{n}+\varepsilon_{m}-\varepsilon_{q}-\varepsilon_{r}\right)\left(\varepsilon_{o}-\varepsilon_{q}\right)\left(\varepsilon_{n}-\varepsilon_{r}\right)}\right] \\
& +\sum_{n>m}^{o c c} \sum_{q>r}^{\text {virt }} \sum_{S}^{\text {virt }} 2 R\left[\{(n q \mid m r)-(n r \mid m q)\} \frac{\left\langle q\left|\hat{h}_{S O}\right| s\right\rangle\left\langle r\left|\hat{h}_{S O}\right| m\right\rangle\left\langle s\left|\hat{h}_{S O}\right| n\right\rangle}{\left(\varepsilon_{n}+\varepsilon_{m}-\varepsilon_{q}-\varepsilon_{r}\right)\left(\varepsilon_{n}-\varepsilon_{s}\right)\left(\varepsilon_{m}-\varepsilon_{r}\right)}\right] \\
& -\sum_{n>m}^{o c c} \sum_{q>r}^{v i r t} \sum_{S}^{v i r t} 2 R\left[\{(n q \mid m r)-(n r \mid m q)\} \frac{\left\langle q\left|\hat{h}_{S O}\right| s\right\rangle\left\langle s\left|\hat{h}_{S O}\right| m\right\rangle\left\langle r\left|\hat{h}_{S O}\right| n\right\rangle}{\left(\varepsilon_{n}+\varepsilon_{m}-\varepsilon_{q}-\varepsilon_{r}\right)\left(\varepsilon_{m}-\varepsilon_{s}\right)\left(\varepsilon_{n}-\varepsilon_{r}\right)}\right] \\
& -\sum_{l>m}^{o c c} \sum_{n}^{o c c} \sum_{q>r}^{v i r t} 2 R\left[\{(l q \mid m r)-(l r \mid m q)\} \frac{\left\langle n\left|\hat{h}_{S O}\right| l\right\rangle\left\langle r\left|\hat{h}_{S O}\right| m\right\rangle\left\langle q\left|\hat{h}_{S O}\right| n\right\rangle}{\left(\varepsilon_{l}+\varepsilon_{m}-\varepsilon_{q}-\varepsilon_{r}\right)\left(\varepsilon_{n}-\varepsilon_{q}\right)\left(\varepsilon_{m}-\varepsilon_{r}\right)}\right] \\
& +\sum_{l>m}^{o c c} \sum_{n}^{o c c} \sum_{q>r}^{\text {virt }} 2 R\left[\{(l q \mid m r)-(l r \mid m q)\} \frac{\left\langle n\left|\hat{h}_{S O}\right| l\right\rangle\left\langle q\left|\hat{h}_{S O}\right| m\right\rangle\left\langle r\left|\hat{h}_{S O}\right| n\right\rangle}{\left(\varepsilon_{l}+\varepsilon_{m}-\varepsilon_{q}-\varepsilon_{r}\right)\left(\varepsilon_{m}-\varepsilon_{q}\right)\left(\varepsilon_{n}-\varepsilon_{r}\right)}\right] \text {. }
\end{aligned}
$$


We can now extend the sums to all values in terms of the indices $m, n, o$ and $p, q, r$ to find:

$$
\begin{aligned}
& E_{D 2}^{(3,1)}=\frac{1}{2} \sum_{m, n}^{o c c} \sum_{p, q, r}^{\text {virt }} R\left[\{(m p \mid n q)-(m q \mid n p)\} \frac{\left\langle q\left|\hat{h}_{S O}\right| n\right\rangle\left\langle p\left|\hat{h}_{S O}\right| r\right\rangle\left\langle r\left|\hat{h}_{S O}\right| m\right\rangle}{\left(\varepsilon_{m}+\varepsilon_{n}-\varepsilon_{p}-\varepsilon_{q}\right)\left(\varepsilon_{m}-\varepsilon_{p}\right)\left(\varepsilon_{m}-\varepsilon_{r}\right)}\right] \\
& -\frac{1}{2} \sum_{m, n, o}^{o c c} \sum_{p, q}^{v i r t} R\left[\{(m p \mid n q)-(m q \mid n p)\} \frac{\left\langle q\left|\hat{h}_{S O}\right| n\right\rangle\left\langle o\left|\hat{h}_{S O}\right| m\right\rangle\left\langle p\left|\hat{h}_{S O}\right| o\right\rangle}{\left(\varepsilon_{m}+\varepsilon_{n}-\varepsilon_{p}-\varepsilon_{q}\right)\left(\varepsilon_{m}-\varepsilon_{p}\right)\left(\varepsilon_{o}-\varepsilon_{p}\right)}\right] \\
& +\frac{1}{2} \sum_{m, n}^{o c c} \sum_{p, q, r}^{v i r t} R\left[\{(m p \mid n q)-(m q \mid n p)\} \frac{\left\langle p\left|\hat{h}_{S O}\right| m\right\rangle\left\langle q\left|\hat{h}_{S O}\right| r\right\rangle\left\langle r\left|\hat{h}_{S O}\right| n\right\rangle}{\left(\varepsilon_{m}+\varepsilon_{n}-\varepsilon_{p}-\varepsilon_{q}\right)\left(\varepsilon_{n}-\varepsilon_{q}\right)\left(\varepsilon_{n}-\varepsilon_{r}\right)}\right] \\
& -\frac{1}{2} \sum_{m, n, o}^{o c c} \sum_{p, q}^{v i r t} R\left[\{(m p \mid n q)-(m q \mid n p)\} \frac{\left\langle p\left|\hat{h}_{S O}\right| m\right\rangle\left\langle o\left|\hat{h}_{S O}\right| n\right\rangle\left\langle q\left|\hat{h}_{S O}\right| o\right\rangle}{\left(\varepsilon_{m}+\varepsilon_{n}-\varepsilon_{p}-\varepsilon_{q}\right)\left(\varepsilon_{n}-\varepsilon_{q}\right)\left(\varepsilon_{o}-\varepsilon_{q}\right)}\right] \\
& -\frac{1}{2} \sum_{m, n}^{\text {occ }} \sum_{p, q, r}^{\text {virt }} R\left[\{(m p \mid n q)-(m q \mid n p)\} \frac{\left\langle q\left|\hat{h}_{S O}\right| m\right\rangle\left\langle p\left|\hat{h}_{S O}\right| r\right\rangle\left\langle r\left|\hat{h}_{S O}\right| n\right\rangle}{\left(\varepsilon_{m}+\varepsilon_{n}-\varepsilon_{p}-\varepsilon_{q}\right)\left(\varepsilon_{n}-\varepsilon_{p}\right)\left(\varepsilon_{n}-\varepsilon_{r}\right)}\right] \\
& +\frac{1}{2} \sum_{m, n, o}^{o c c} \sum_{p, q}^{v i r t} R\left[\{(m p \mid n q)-(m q \mid n p)\} \frac{\left\langle q\left|\hat{h}_{S O}\right| m\right\rangle\left\langle o\left|\hat{h}_{S O}\right| n\right\rangle\left\langle p\left|\hat{h}_{S O}\right| o\right\rangle}{\left(\varepsilon_{m}+\varepsilon_{n}-\varepsilon_{p}-\varepsilon_{q}\right)\left(\varepsilon_{n}-\varepsilon_{p}\right)\left(\varepsilon_{o}-\varepsilon_{p}\right)}\right] \\
& -\frac{1}{2} \sum_{m, n}^{\text {occ }} \sum_{p, q, r}^{\text {virt }} R\left[\{(m p \mid n q)-(m q \mid n p)\} \frac{\left\langle p\left|\hat{h}_{S O}\right| n\right\rangle\left\langle q\left|\hat{h}_{S O}\right| r\right\rangle\left\langle r\left|\hat{h}_{S O}\right| m\right\rangle}{\left(\varepsilon_{m}+\varepsilon_{n}-\varepsilon_{p}-\varepsilon_{q}\right)\left(\varepsilon_{m}-\varepsilon_{q}\right)\left(\varepsilon_{m}-\varepsilon_{r}\right)}\right] \\
& +\frac{1}{2} \sum_{m, n, o}^{o c c} \sum_{p, q}^{v i r t} R\left[\{(m p \mid n q)-(m q \mid n p)\} \frac{\left\langle p\left|\hat{h}_{S O}\right| n\right\rangle\left\langle o\left|\hat{h}_{S O}\right| m\right\rangle\left\langle q\left|\hat{h}_{S O}\right| o\right\rangle}{\left(\varepsilon_{m}+\varepsilon_{n}-\varepsilon_{p}-\varepsilon_{q}\right)\left(\varepsilon_{m}-\varepsilon_{q}\right)\left(\varepsilon_{o}-\varepsilon_{q}\right)}\right] \\
& +\frac{1}{2} \sum_{m, n}^{\text {occ }} \sum_{p, q, r}^{\text {virt }} R\left[\{(n p \mid m q)-(n q \mid m p)\} \frac{\left\langle q\left|\hat{h}_{S O}\right| r\right\rangle\left\langle r\left|\hat{h}_{S O}\right| m\right\rangle\left\langle p\left|\hat{h}_{S O}\right| n\right\rangle}{\left(\varepsilon_{n}+\varepsilon_{m}-\varepsilon_{p}-\varepsilon_{q}\right)\left(\varepsilon_{m}-\varepsilon_{r}\right)\left(\varepsilon_{n}-\varepsilon_{p}\right)}\right] \\
& -\frac{1}{2} \sum_{m, n}^{o c c} \sum_{p, q, r}^{v i r t} R\left[\{(n p \mid m q)-(n q \mid m p)\} \frac{\left\langle q\left|\hat{h}_{S O}\right| r\right\rangle\left\langle p\left|\hat{h}_{S O}\right| m\right\rangle\left\langle r\left|\hat{h}_{S O}\right| n\right\rangle}{\left(\varepsilon_{n}+\varepsilon_{m}-\varepsilon_{p}-\varepsilon_{q}\right)\left(\varepsilon_{m}-\varepsilon_{p}\right)\left(\varepsilon_{n}-\varepsilon_{r}\right)}\right] \\
& -\frac{1}{2} \sum_{m, n, o}^{o c c} \sum_{p, q}^{v i r t} R\left[\{(n p \mid m q)-(n q \mid m p)\} \frac{\left\langle o\left|\hat{h}_{S O}\right| m\right\rangle\left\langle q\left|\hat{h}_{S O}\right| o\right\rangle\left\langle p\left|\hat{h}_{S O}\right| n\right\rangle}{\left(\varepsilon_{n}+\varepsilon_{m}-\varepsilon_{p}-\varepsilon_{q}\right)\left(\varepsilon_{n}-\varepsilon_{p}\right)\left(\varepsilon_{o}-\varepsilon_{q}\right)}\right] \\
& +\frac{1}{2} \sum_{m, n, o}^{o c c} \sum_{p, q}^{v i r t} R\left[\{(n p \mid m q)-(n q \mid m p)\} \frac{\left\langle o\left|\hat{h}_{S O}\right| m\right\rangle\left\langle p\left|\hat{h}_{S O}\right| o\right\rangle\left\langle q\left|\hat{h}_{S O}\right| n\right\rangle}{\left(\varepsilon_{n}+\varepsilon_{m}-\varepsilon_{p}-\varepsilon_{q}\right)\left(\varepsilon_{o}-\varepsilon_{p}\right)\left(\varepsilon_{n}-\varepsilon_{q}\right)}\right] \\
& +\frac{1}{2} \sum_{m, n}^{\text {occ }} \sum_{p, q, r}^{\text {virt }} R\left[\{(n p \mid m q)-(n q \mid m p)\} \frac{\left\langle p\left|\hat{h}_{S O}\right| r\right\rangle\left\langle q\left|\hat{h}_{S O}\right| m\right\rangle\left\langle r\left|\hat{h}_{S O}\right| n\right\rangle}{\left(\varepsilon_{n}+\varepsilon_{m}-\varepsilon_{p}-\varepsilon_{q}\right)\left(\varepsilon_{n}-\varepsilon_{r}\right)\left(\varepsilon_{m}-\varepsilon_{q}\right)}\right] \\
& -\frac{1}{2} \sum_{m, n}^{\text {occ }} \sum_{p, q, r}^{\text {virt }} R\left[\{(n p \mid m q)-(n q \mid m p)\} \frac{\left\langle p\left|\hat{h}_{S O}\right| r\right\rangle\left\langle r\left|\hat{h}_{S O}\right| m\right\rangle\left\langle q\left|\hat{h}_{S O}\right| n\right\rangle}{\left(\varepsilon_{n}+\varepsilon_{m}-\varepsilon_{p}-\varepsilon_{q}\right)\left(\varepsilon_{m}-\varepsilon_{r}\right)\left(\varepsilon_{n}-\varepsilon_{q}\right)}\right] \\
& -\frac{1}{2} \sum_{m, n, o}^{o c c} \sum_{p, q}^{\text {virt }} R\left[\{(n p \mid m q)-(n q \mid m p)\} \frac{\left\langle o\left|\hat{h}_{S O}\right| n\right\rangle\left\langle q\left|\hat{h}_{S O}\right| m\right\rangle\left\langle p\left|\hat{h}_{S O}\right| o\right\rangle}{\left(\varepsilon_{n}+\varepsilon_{m}-\varepsilon_{p}-\varepsilon_{q}\right)\left(\varepsilon_{o}-\varepsilon_{p}\right)\left(\varepsilon_{m}-\varepsilon_{q}\right)}\right] \\
& +\frac{1}{2} \sum_{m, n, o}^{o c c} \sum_{p, q}^{\text {virt }} R\left[\{(n p \mid m q)-(n q \mid m p)\} \frac{\left\langle o\left|\hat{h}_{S O}\right| n\right\rangle\left\langle p\left|\hat{h}_{S O}\right| m\right\rangle\left\langle q\left|\hat{h}_{S O}\right| o\right\rangle}{\left(\varepsilon_{n}+\varepsilon_{m}-\varepsilon_{p}-\varepsilon_{q}\right)\left(\varepsilon_{m}-\varepsilon_{p}\right)\left(\varepsilon_{o}-\varepsilon_{q}\right)}\right] \text {. }
\end{aligned}
$$

Combining i) the first, third (after a switch of the indices $m \leftrightarrow n$ and $p \leftrightarrow q$ ), fifth (after a switch $n \leftrightarrow m$ ), and seventh (after a switch $p \leftrightarrow q$ ) lines of Eq. (S42) ii) the second, fourth (after a switch of the indices $m \leftrightarrow n$ and $p \leftrightarrow q$ ), sixth (after a switch $m \leftrightarrow n$ ), and eighth (after a switch $p \leftrightarrow q$ ) lines of Eq. (S42) iii) the ninth, tenth (after a switch of the indices $m \leftrightarrow n$ ), tirteenth (after a switch $m \leftrightarrow n$ and $p \leftrightarrow q$ ), and fourteenth (after a switch $p \leftrightarrow q$ ) lines of Eq. (S42) iv) the eleventh, twelfth (after a 
switch of the indices $p \leftrightarrow q$ ), fifteenth (after a switch $m \leftrightarrow n$ and $p \leftrightarrow q$ ), and sixteenth (after a switch $m \leftrightarrow n$ ), we obtain:

$$
\begin{aligned}
E_{D 2}^{(3,1)} & =2 \sum_{m, n}^{o c c} \sum_{p, q, r}^{\text {virt }} R\left[\{(m p \mid n q)-(m q \mid n p)\} \frac{\left\langle q\left|\hat{h}_{S O}\right| n\right\rangle\left\langle p\left|\hat{h}_{S O}\right| r\right\rangle\left\langle r\left|\hat{h}_{S O}\right| m\right\rangle}{\left(\varepsilon_{m}+\varepsilon_{n}-\varepsilon_{p}-\varepsilon_{q}\right)\left(\varepsilon_{m}-\varepsilon_{p}\right)\left(\varepsilon_{m}-\varepsilon_{r}\right)}\right] \\
& -2 \sum_{m, n, o}^{o c c} \sum_{p, q}^{\text {virt }} R\left[\{(m p \mid n q)-(m q \mid n p)\} \frac{\left\langle q\left|\hat{h}_{S O}\right| n\right\rangle\left\langle o\left|\hat{h}_{S O}\right| m\right\rangle\left\langle p\left|\hat{h}_{S O}\right| o\right\rangle}{\left(\varepsilon_{m}+\varepsilon_{n}-\varepsilon_{p}-\varepsilon_{q}\right)\left(\varepsilon_{m}-\varepsilon_{p}\right)\left(\varepsilon_{o}-\varepsilon_{p}\right)}\right] \\
& +2 \sum_{m, n}^{o c c} \sum_{,, q, r}^{\text {virt }} R\left[\{(n p \mid m q)-(n q \mid m p)\} \frac{\left\langle q\left|\hat{h}_{S O}\right| r\right\rangle\left\langle r\left|\hat{h}_{S O}\right| m\right\rangle\left\langle p\left|\hat{h}_{S O}\right| n\right\rangle}{\left(\varepsilon_{n}+\varepsilon_{m}-\varepsilon_{p}-\varepsilon_{q}\right)\left(\varepsilon_{m}-\varepsilon_{r}\right)\left(\varepsilon_{n}-\varepsilon_{p}\right)}\right] \\
& -2 \sum_{m, n, o}^{o c c} \sum_{p, q}^{\text {virt }} R\left[\{(n p \mid m q)-(n q \mid m p)\} \frac{\left\langle o\left|\hat{h}_{S O}\right| m\right\rangle\left\langle q\left|\hat{h}_{S O}\right| o\right\rangle\left\langle p\left|\hat{h}_{S O}\right| n\right\rangle}{\left(\varepsilon_{n}+\varepsilon_{m}-\varepsilon_{p}-\varepsilon_{q}\right)\left(\varepsilon_{n}-\varepsilon_{p}\right)\left(\varepsilon_{o}-\varepsilon_{q}\right)}\right] .
\end{aligned}
$$

Switching the indices $p \leftrightarrow q$ in lines 3 and 4 of Eq. (S43) and combining them with lines 1 and 2 gives:

$$
\begin{aligned}
E_{D 2}^{(3,1)} & =2 \sum_{m, n}^{o c c} \sum_{p, q, r}^{\text {virt }} R\left[\{(m p \mid n q)-(m q \mid n p)\} \frac{\left\langle q\left|\hat{h}_{S O}\right| n\right\rangle\left\langle p\left|\hat{h}_{S O}\right| r\right\rangle\left\langle r\left|\hat{h}_{S O}\right| m\right\rangle}{\left(\varepsilon_{m}+\varepsilon_{n}-\varepsilon_{p}-\varepsilon_{q}\right)}\right]\left[\frac{1}{\left(\varepsilon_{m}-\varepsilon_{p}\right)\left(\varepsilon_{m}-\varepsilon_{r}\right)}+\frac{1}{\left(\varepsilon_{n}-\varepsilon_{q}\right)\left(\varepsilon_{m}-\varepsilon_{r}\right)}\right] \\
& -2 \sum_{m, n, o}^{o c c} \sum_{p, q}^{\text {virt }} R\left[\{(m p \mid n q)-(m q \mid n p)\} \frac{\left\langle q\left|\hat{h}_{S O}\right| n\right\rangle\left\langle o\left|\hat{h}_{S O}\right| m\right\rangle\left\langle p\left|\hat{h}_{S O}\right| o\right\rangle}{\left(\varepsilon_{m}+\varepsilon_{n}-\varepsilon_{p}-\varepsilon_{q}\right)}\right]\left[\frac{1}{\left(\varepsilon_{m}-\varepsilon_{p}\right)\left(\varepsilon_{o}-\varepsilon_{p}\right)}+\frac{1}{\left(\varepsilon_{n}-\varepsilon_{q}\right)\left(\varepsilon_{o}-\varepsilon_{p}\right)}\right] \mathrm{S}
\end{aligned}
$$

Finally expressing both terms of lines 1 and 2 of Eq. (S44) with a common denominator, we obtain:

$$
\begin{aligned}
E_{D 2}^{(3,1)} & =2 \sum_{m, n}^{o c c} \sum_{p, q, r}^{\text {virt }} R\left[\{(m p \mid n q)-(m q \mid n p)\} \frac{\left\langle q\left|\hat{h}_{S O}\right| n\right\rangle\left\langle p\left|\hat{h}_{S O}\right| r\right\rangle\left\langle r\left|\hat{h}_{S O}\right| m\right\rangle}{\left(\varepsilon_{n}-\varepsilon_{q}\right)\left(\varepsilon_{m}-\varepsilon_{p}\right)\left(\varepsilon_{m}-\varepsilon_{r}\right)}\right] \\
& -2 \sum_{m, n, o}^{o c c} \sum_{p, q}^{\text {virt }} R\left[\{(m p \mid n q)-(m q \mid n p)\} \frac{\left\langle q\left|\hat{h}_{S O}\right| n\right\rangle\left\langle o\left|\hat{h}_{S O}\right| m\right\rangle\left\langle p\left|\hat{h}_{S O}\right| o\right\rangle}{\left(\varepsilon_{n}-\varepsilon_{q}\right)\left(\varepsilon_{m}-\varepsilon_{p}\right)\left(\varepsilon_{o}-\varepsilon_{p}\right)}\right] .
\end{aligned}
$$

Combining Eqs. (S35) and (S45) we obtain Eq. (B20).

\section{FORMULA FOR $E^{(3,1)}$ IN THE BASIS OF ATOMIC ORBITALS}

From Eq. (S23), we have for $E_{S}^{(3,1)}$ :

$$
E_{S}^{(3,1)}=\sum_{m, n}^{o c c} \sum_{p, q}^{v i r t}[(p m \mid n q)-(p q \mid n m)]\left[\sum_{r}^{v i r t} \frac{\left\langle m\left|\hat{h}_{S O}\right| p\right\rangle\left\langle q\left|\hat{h}_{S O}\right| r\right\rangle\left\langle r\left|\hat{h}_{S O}\right| n\right\rangle}{\left(\varepsilon_{m}-\varepsilon_{p}\right)\left(\varepsilon_{n}-\varepsilon_{q}\right)\left(\varepsilon_{n}-\varepsilon_{r}\right)}-\sum_{o}^{o c c} \frac{\left\langle m\left|\hat{h}_{S O}\right| p\right\rangle\left\langle o\left|\hat{h}_{S O}\right| n\right\rangle\left\langle q\left|\hat{h}_{S O}\right| o\right\rangle}{\left(\varepsilon_{m}-\varepsilon_{p}\right)\left(\varepsilon_{n}-\varepsilon_{q}\right)\left(\varepsilon_{o}-\varepsilon_{q}\right)}\right]+\text { c.c. }
$$

where c.c. denotes the complex-conjugate. Then, substituting Eqs. (15b) and (B21a) in Eq. (S46) gives:

$$
E_{S}^{(3,1)}=\sum_{m, n}^{o c c} \sum_{p, q}^{v i r t}[(p m \mid n q)-(p q \mid n m)] U_{m p}^{(1,0)} U_{q n}^{(2,0)}+\text { c.c. . }
$$

Upon substituting of Eq. (B15) in Eq. (S47), we obtain the computationally convenient formula:

$$
\begin{aligned}
E_{S}^{(3,1)} & =\sum_{\sigma \sigma^{\prime}} \sum_{\omega v}\left[M_{\omega v}^{\sigma \sigma(1,0)}\right]^{*} \sum_{\tau \gamma} M_{\gamma \tau}^{\sigma^{\prime} \sigma^{\prime}(2,0)}(\omega v \mid \tau \gamma) \\
& -\sum_{\sigma \sigma^{\prime}} \sum_{\omega v}\left[M_{\omega v}^{\sigma \sigma^{\prime}(1,0)}\right]^{*} \sum_{\tau \gamma} M_{\gamma \tau}^{\sigma \sigma^{\prime}(2,0)}(\omega \gamma \mid \tau \nu)+\text { c.c. }
\end{aligned}
$$

For $E_{D 2}^{(3,1)}$, we switch the indices $q, n \leftrightarrow p, m$ in Eq. (S45), which yields:

$$
E_{D 2}^{(3,1)}=\sum_{m, n}^{o c c} \sum_{p, q}^{\text {virt }}\{(m p \mid n q)-(m q \mid n p)\}\left[\sum_{r}^{\text {virt }} \frac{\left\langle p\left|\hat{h}_{S O}\right| m\right\rangle\left\langle q\left|\hat{h}_{S O}\right| r\right\rangle\left\langle r\left|\hat{h}_{S O}\right| n\right\rangle}{\left(\varepsilon_{n}-\varepsilon_{q}\right)\left(\varepsilon_{m}-\varepsilon_{p}\right)\left(\varepsilon_{n}-\varepsilon_{r}\right)}-\sum_{o}^{o c c} \frac{\left\langle p\left|\hat{h}_{S O}\right| m\right\rangle\left\langle o\left|\hat{h}_{S O}\right| n\right\rangle\left\langle q\left|\hat{h}_{S O}\right| o\right\rangle}{\left(\varepsilon_{n}-\varepsilon_{q}\right)\left(\varepsilon_{m}-\varepsilon_{p}\right)\left(\varepsilon_{o}-\varepsilon_{q}\right)}\right]+\text { c.c. . }
$$


Then, substituting Eqs. (15b) and (B21a) in Eq. (S49) we find:

$$
E_{D 2}^{(3,1)}=\sum_{m, n}^{o c c} \sum_{p, q}^{\text {virt }}[(m p \mid n q)-(m q \mid n p)] U_{p m}^{(1,0)} U_{q n}^{(2,0)}+\text { c.c. . }
$$

Now substituting Eq. (B15) in Eq. (S50), we get:

$$
\begin{aligned}
E_{D 2}^{(3,1)} & =\sum_{\sigma \sigma^{\prime} \omega v} \sum_{v \omega}^{\sigma \sigma(1,0)} \sum_{\tau \gamma} M_{\gamma \tau}^{\sigma^{\prime} \sigma^{\prime}(2,0)}(\omega v \mid \tau \gamma) \\
& -\sum_{\sigma \sigma^{\prime} \omega v} \sum_{\nu \omega}^{\sigma^{\prime} \sigma(1,0)} \sum_{\tau \gamma} M_{\gamma \tau}^{\sigma \sigma^{\prime}(2,0)}(\omega \gamma \mid \tau \nu)+\text { c.c. }
\end{aligned}
$$

For $E_{D 1}^{(3,1)}$, we switch the indices $q, n \leftrightarrow p, m$ in Eq. (S35), and obtain:

$$
\begin{aligned}
E_{D 1}^{(3,1)} & =\sum_{m, n}^{o c c} \sum_{p, q, r}^{\text {virt }}\{(m p \mid r q)-(m q \mid r p)\} \frac{\left\langle n\left|\hat{h}_{S O}\right| r\right\rangle\left\langle q\left|\hat{h}_{S O}\right| n\right\rangle\left\langle p\left|\hat{h}_{S O}\right| m\right\rangle}{\left(\varepsilon_{n}-\varepsilon_{r}\right)\left(\varepsilon_{n}-\varepsilon_{q}\right)\left(\varepsilon_{m}-\varepsilon_{p}\right)} \\
& -\sum_{m, n, o}^{o c c} \sum_{p, q}^{v i r t}\{(m p \mid n o)-(m o \mid n p)\} \frac{\left\langle o\left|\hat{h}_{S O}\right| q\right\rangle\left\langle q \mid \hat{h}_{S O} n\right\rangle\left\langle p\left|\hat{h}_{S O}\right| m\right\rangle}{\left(\varepsilon_{o}-\varepsilon_{q}\right)\left(\varepsilon_{n}-\varepsilon_{q}\right)\left(\varepsilon_{m}-\varepsilon_{p}\right)}+\text { c.c. }
\end{aligned}
$$

and substituting Eq. (15b) in Eq. (S52), we find:

$$
\begin{aligned}
E_{D 1}^{(3,1)} & =\sum_{m, n}^{o c c} \sum_{p, q, r}^{\text {virt }}\{(m p \mid r q)-(m q \mid r p)\} U_{p m}^{(1,0)} \frac{\left\langle n\left|\hat{h}_{S O}\right| r\right\rangle\left\langle q\left|\hat{h}_{S O}\right| n\right\rangle}{\left(\varepsilon_{n}-\varepsilon_{r}\right)\left(\varepsilon_{n}-\varepsilon_{q}\right)} \\
& -\sum_{m, n, o}^{o c c} \sum_{p, q}^{v i r t}\{(m p \mid n o)-(m o \mid n p)\} U_{p m}^{(1,0)} \frac{\left\langle o\left|\hat{h}_{S O}\right| q\right\rangle\left\langle q \mid \hat{h}_{S O} n\right\rangle}{\left(\varepsilon_{o}-\varepsilon_{q}\right)\left(\varepsilon_{n}-\varepsilon_{q}\right)}+\text { c.c. . }
\end{aligned}
$$

Introducing the occ-occ and virt-virt blocks of $\mathbf{U}^{(2,0)}$ :

$$
U_{q r}^{\sigma \sigma^{\prime}(2,0)}=-\frac{1}{2} \sum_{\sigma^{\prime \prime}} \sum_{n}^{o c c}\left[U_{n q}^{\sigma^{\prime \prime} \sigma(1,0)}\right]^{*} U_{n r}^{\sigma^{\prime \prime} \sigma^{\prime}(1,0)}
$$

and:

$$
U_{o n}^{\sigma \sigma^{\prime}(2,0)}=-\frac{1}{2} \sum_{\sigma^{\prime \prime}} \sum_{q}^{v i r t}\left[U_{q o}^{\sigma^{\prime \prime} \sigma(1,0)}\right]^{*} U_{q n}^{\sigma^{\prime \prime} \sigma^{\prime}(1,0)}
$$

then, substituting Eq. (S54) in Eq. (S53) yields:

$$
E_{D 1}^{(3,1)}=-2 \sum_{m}^{o c c} \sum_{p, q, r}^{v i r t}\{(m p \mid r q)-(m q \mid r p)\} U_{p m}^{(1,0)} U_{q r}^{(2,0)}+2 \sum_{m, n, o}^{o c c} \sum_{p}^{v i r t}\{(m p \mid n o)-(m o \mid n p)\} U_{p m}^{(1,0)} U_{o n}^{(2,0)}+\text { c.c. . }
$$

Finally, using the matrices:

$$
O_{\omega v}^{\sigma \sigma^{\prime}(2,0)}=\sum_{o n}^{o c c} c_{\omega o}^{\sigma}\left[c_{v n}^{\sigma^{\prime}}\right]^{*} U_{o n}^{\sigma \sigma^{\prime}(2,0)}
$$

and:

$$
V_{\omega v}^{\sigma \sigma^{\prime}(2,0)}=\sum_{q r}^{v i r t} c_{\omega q}^{\sigma}\left[c_{v r}^{\sigma^{\prime}}\right]^{*} U_{q r}^{\sigma \sigma^{\prime}(2,0)}
$$

then substituting Eqs. (S56) and (B15) in Eq. (S55), we get:

$$
\begin{aligned}
E_{D 1}^{(3,1)} & =2 \sum_{\sigma \sigma^{\prime} \omega v} \sum_{v \omega}^{\sigma \sigma(1,0)} \sum_{\tau \gamma}\left[O_{\gamma \tau}^{\sigma^{\prime} \sigma^{\prime}(2,0)}-V_{\gamma \tau}^{\sigma^{\prime} \sigma^{\prime}(2,0)}\right](\omega v \mid \tau \gamma) \\
& -2 \sum_{\sigma \sigma^{\prime} \omega v} \sum_{v \omega}^{\sigma^{\prime} \sigma(1,0)} \sum_{\tau \gamma}\left[O_{\gamma \tau}^{\sigma \sigma^{\prime}(2,0)}-V_{\gamma \tau}^{\sigma \sigma^{\prime}(2,0)}\right](\omega \gamma \mid \tau \nu)+\text { c.c. }
\end{aligned}
$$


Taking the sum of Eqs. (S48), (S51) and (S57), and using Eq. (B17) we finally obtain:

$$
\begin{aligned}
E^{(3,1)} & =2 \sum_{\sigma \sigma^{\prime}} R\left[\sum_{\omega \nu}\left[P_{\omega v}^{\sigma \sigma(1,0)}\right]^{*} \sum_{\tau \gamma} M_{\gamma \tau}^{\sigma^{\prime} \sigma^{\prime}(2,0)}(\omega v \mid \tau \gamma)\right]-2 \sum_{\sigma \sigma^{\prime}} R\left[\sum_{\omega \nu}\left[P_{\omega \nu}^{\sigma \sigma^{\prime}(1,0)}\right]^{*} \sum_{\tau \gamma} M_{\gamma \tau}^{\sigma \sigma^{\prime}(2,0)}(\omega \gamma \mid \tau v)\right] \\
& +4 \sum_{\sigma \sigma^{\prime}} R\left[\sum_{\omega \nu} M_{v \omega}^{\sigma \sigma(1,0)} \sum_{\tau \gamma}\left[O_{\gamma \tau}^{\sigma^{\prime} \sigma^{\prime}(2,0)}-V_{\gamma \tau}^{\sigma^{\prime} \sigma^{\prime}(2,0)}\right](\omega v \mid \tau \gamma)\right] \\
& -4 \sum_{\sigma \sigma^{\prime}} R\left[\sum_{\omega \nu} M_{v \omega}^{\sigma^{\prime} \sigma(1,0)} \sum_{\tau \gamma}\left[O_{\gamma \tau}^{\sigma \sigma^{\prime}(2,0)}-V_{\gamma \tau}^{\sigma \sigma^{\prime}(2,0)}\right](\omega \gamma \mid \tau \nu)\right] .
\end{aligned}
$$

Eq. (S58) is identical to Eq. (23) of the main text.

\section{COMPUTATIONAL RESULTS ON SEO AND TS TS $^{+}$}

TABLE S1: Same as the tables of the main text, except that calculations are performed on the SeO and $\mathrm{Ts}_{2}^{+}$molecules (with the Stuttgart potentials and double-zeta valence basis sets).

\begin{tabular}{rcc}
\hline \hline & $\mathrm{SeO}$ & $\mathrm{Ts}_{2}^{+}$ \\
& & \\
$E^{(2,0)}$ & $-3.69 \times 10^{-01}$ & $-1.41 \times 10^{+00}$ \\
$E^{(3,0)}$ & $+3.29 \times 10^{-02}$ & $-4.59 \times 10^{-02}$ \\
$E^{(4,0)}$ & $+7.85 \times 10^{-03}$ & $+5.05 \times 10^{-02}$ \\
$E^{(2,1)}$ & $-1.27 \times 10^{-01}$ & $-3.33 \times 10^{-01}$ \\
$E^{(3,1)}$ & $+5.96 \times 10^{-03}$ & $+7.37 \times 10^{-02}$ \\
& & \\
& \\
$\mathrm{PT} \infty$ & $-3.29 \times 10^{-01}$ & $-1.41 \times 10^{+00}$ \\
$\mathrm{PT} \infty 1$ & $-4.50 \times 10^{-01}$ & $-1.67 \times 10^{+00}$ \\
$\mathrm{SV}$ & $-4.51 \times 10^{-01}$ & $-1.49 \times 10^{+00}$ \\
$\Delta_{21}$ & $-4.56 \times 10^{-02}$ & $-2.54 \times 10^{-01}$ \\
$\Delta_{\infty 1}$ & $+9.34 \times 10^{-04}$ & $-1.78 \times 10^{-01}$ \\
\hline \hline
\end{tabular}

TABLE S2: Same as the tables of the main text, except that calculations are performed on the positively charged halogen diatomic molecules

\begin{tabular}{|c|c|c|c|c|c|c|}
\hline & $\mathrm{Br}_{2}^{+}$ & $\mathrm{I}_{2}^{+}$ & $\mathrm{At}_{2}^{+}$ & $\mathrm{Ts}_{2}^{+}$ & $0.90 \times \mathrm{Ts}_{2}^{+}$ & $0.91 \times \mathrm{Ts}_{2}^{+}$ \\
\hline$E^{(2,0)}$ & $-2.92 \times 10^{-03}$ & $-4.73 \times 10^{-03}$ & $-3.39 \times 10^{-02}$ & $-1.41 \times 10^{+00}$ & $-1.14 \times 10^{+00}$ & $-1.17 \times 10^{+00}$ \\
\hline$E^{(3,0)}$ & $+3.07 \times 10^{-05}$ & $-3.54 \times 10^{-05}$ & $-1.50 \times 10^{-03}$ & $-4.59 \times 10^{-02}$ & $-3.35 \times 10^{-02}$ & $-3.46 \times 10^{-02}$ \\
\hline$E^{(4,0)}$ & $-3.34 \times 10^{-06}$ & $-4.86 \times 10^{-06}$ & $+4.85 \times 10^{-05}$ & $+5.05 \times 10^{-02}$ & $+3.31 \times 10^{-02}$ & $+3.46 \times 10^{-02}$ \\
\hline$E^{(2,1)}$ & $-5.23 \times 10^{-04}$ & $-2.88 \times 10^{-03}$ & $-1.41 \times 10^{-02}$ & $-3.33 \times 10^{-01}$ & $-2.70 \times 10^{-01}$ & $-2.76 \times 10^{-01}$ \\
\hline$E^{(3,1)}$ & $-1.01 \times 10^{-05}$ & $+7.82 \times 10^{-05}$ & $-9.28 \times 10^{-04}$ & $+7.37 \times 10^{-02}$ & $+5.37 \times 10^{-02}$ & $+5.55 \times 10^{-02}$ \\
\hline $\mathrm{PT} \infty$ & $-2.89 \times 10^{-03}$ & $-4.76 \times 10^{-03}$ & $-3.54 \times 10^{-02}$ & $-1.41 \times 10^{+00}$ & $-1.14 \times 10^{+00}$ & $-1.17 \times 10^{+00}$ \\
\hline$P T \infty 1$ & $-3.42 \times 10^{-03}$ & $-7.56 \times 10^{-03}$ & $-5.04 \times 10^{-02}$ & $-1.67 \times 10^{+00}$ & $-1.36 \times 10^{+00}$ & $-1.39 \times 10^{+00}$ \\
\hline SV & $-3.41 \times 10^{-03}$ & $-7.48 \times 10^{-03}$ & $-4.99 \times 10^{-02}$ & $-1.49 \times 10^{+00}$ & $-1.23 \times 10^{+00}$ & $-1.25 \times 10^{+00}$ \\
\hline$\Delta_{21}$ & $-2.59 \times 10^{-05}$ & $-1.26 \times 10^{-04}$ & $+1.82 \times 10^{-03}$ & $-2.54 \times 10^{-01}$ & $-1.85 \times 10^{-01}$ & $-1.91 \times 10^{-01}$ \\
\hline$\Delta_{\infty 1}$ & $-8.64 \times 10^{-06}$ & $-7.87 \times 10^{-05}$ & $-5.57 \times 10^{-04}$ & $-1.78 \times 10^{-01}$ & $-1.33 \times 10^{-01}$ & $-1.37 \times 10^{-01}$ \\
\hline
\end{tabular}
(with the Stuttgart potentials). The last two rows are for the system $\mathrm{Ts}_{2}^{+}$, with the SOREP operator scaled down by factors of 0.90 and 0.91 , respectively. 


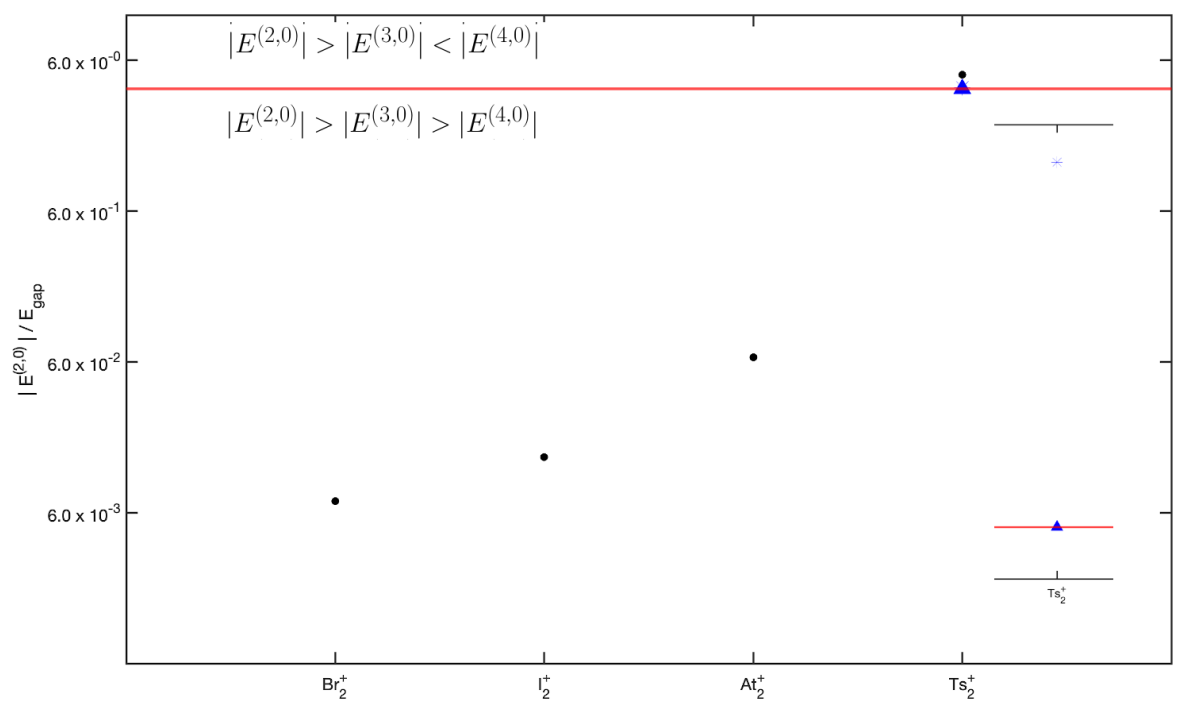

FIG. S1: Ratio of $\left|E^{(2,0)}\right|$ over the HOMO-LUMO gap (plotted on a log scale) for the series $\mathrm{Br}_{2}^{+}$to $\mathrm{Ts}_{2}^{+}$(black circles), for the calculations of Table S2. The blue triangle and asterisk (also shown in close-up panel) are for $\mathrm{Ts}_{2}^{+}$with the SOC operator scaled down by a factor of 0.90 and 0.91 , respectively. 